\title{
A historical account of types of fuzzy sets and their relationships
}

\author{
Humberto Bustince, Senior Member, IEEE, Edurne Barrenechea, Miguel Pagola, Javier Fernandez, \\ Zeshui Xu, Senior Member, IEEE, \\ Benjamin Bedregal, Javier Montero, Hani Hagras, Fellow, IEEE, \\ Francisco Herrera, Member, IEEE, and Bernard De Baets, Member, IEEE.
}

\begin{abstract}
In this work we review the definition and basic properties of the different types of fuzzy sets that have appeared up to now in the literature. We also analyze the relationships between them and enumerate some of the applications in which they have been used.
\end{abstract}

Index Terms-Type-2 fuzzy set; Set-valued fuzzy set; Hesitant fuzzy set; Interval-valued fuzzy set; Atanassov intuitionistic fuzzy set; Interval type-2 fuzzy sets; Interval-valued Atanassov intuitionistic fuzzy set; Neutrosophic set; Bipolar-valued fuzzy set; Fuzzy multiset; Fuzzy rough set; Fuzzy soft set; Multi-polarvalued fuzzy set.

\section{INTRODUCTION}

It has been widely accepted, from the definition of Fuzzy Sets (FSs) in 1965 [163] and its generalization by Goguen in 1967 [61] ( $L$-FSs), that the main obstacle in their application is the attribution of membership degrees to the elements, since these depend on the application and the context. For this reason, Zadeh [164], [165] elaborated on the fact that in fuzzy logic everything is allowed to be a matter of degree (where the degree could be fuzzy). Hence, in 1971 [164], Zadeh presented the concept of Type-n Fuzzy Sets (TnFSs), which includes Type-2 Fuzzy Sets (T2FSs).

Since 1971, several different types of FSs have been introduced, some of them aimed at solving the problem of constructing the membership degrees of the elements to the FS, and others focused on representing the uncertainty linked to the considered problem in a way different from the one proposed by Zadeh.

H. Bustince, E. Barrenechea, M. Pagola, J. Fernandez are with the Departamento de Automática y Computación, Universidad Pública de Navarra, Campus Arrosadia s/n, P.O. Box 31006, Pamplona, Spain.

H. Bustince, E. Barrenechea are with the Institute of Smart Cities, Universidad Publica de Navarra, Campus Arrosadia s/n, 31006 Pamplona, Spain.

Z. S. Xu is with the Business School, Sichuan University, Chengdu 610064 , China.

B. Bedregal is with the Departamento de Informática e Matemática Aplicada, Universidade Federal do Rio Grande do Norte, Campus Universitário s/n, 59072-970 Natal, Brazil.

J. Montero is with the Facultad de Ciencias Matemáticas, Universidad Complutense, Plaza de las Ciencias 3,28040 Madrid, Spain.

H. Hagras is with the The Computational Intelligence Centre, School of Computer Science and Electronic Engineering, University of Essex, Wivenhoe Park, Colchester, CO43SQ, UK.

F. Herrera is with the Department of Computation and Artificial Intelligence, CITIC-UGR, Universidad de Granada, Spain and King Abdulaziz Univ, Fac Comp \& Informat Technol North Jeddah, Jeddah 21589, Saudi Arabia.

B. De Baets is with the Department of Mathematical Modelling, Statistics and Bioinformatics, Ghent University, Coupure links 653, B-9000 Gent, Belgium
In Table I a historical sequence of the appearance of the different types of fuzzy sets is displayed. In Table II we present a list of the acronyms.

TABLE II

NAMES AND ACRONYMS

\begin{tabular}{l|l} 
Name & Acronym \\
\hline \hline Atanassov Intuitionistic Fuzzy Sets & AIFSs \\
Bipolar-Valued Fuzzy Sets of Lee & BVFSLs \\
Bipolar-Valued Fuzzy Sets of Zhang & BVFSZs \\
Complex Fuzzy Sets & CFSs \\
Fuzzy Sets & FSs \\
Fuzzy Rough Sets & FRSs \\
Fuzzy Soft Sets & FSSs \\
Grey Sets & GSs \\
Hesitant Fuzzy Sets & HFSs \\
Interval Type-2 Fuzzy Sets & IT2FSs \\
Interval-Valued Atanassov & \\
\multicolumn{1}{c}{ Intuitionistic Fuzzy Sets } & IVAIFSs \\
Interval-Valued Fuzzy Sets & IVFSs \\
m-Polar-Valued Fuzzy Sets & mPVFSs \\
Neutrosophic Sets & NSs \\
Pythagorean Fuzzy Sets & PFSs \\
Set-Valued Fuzzy Sets & SVFSs \\
Shadow Sets & SSs \\
Type-2 Fuzzy Sets & T2FFs \\
Type-n Fuzzy Sets & TnFSs \\
Typical Hesitant Fuzzy Sets & THFSs \\
Vague Sets & VSs \\
\hline
\end{tabular}

Related to Table I, the goals of this work are the following:

1.- To introduce the definition and basic properties of each of the types of FSs.

2.- To study the relationships between the different types of fuzzy sets. In particular, to show that Hesitant Fuzzy Sets (HFSs) are, conceptually, the same as Set-Valued Fuzzy Sets (SVFSs), as defined by Grattan-Guinness. However, Torra provided an explicit definition for union and intersection of HFSs, whereas this was not the case for Grattan-Guinness' SVFSs.

3.- To analyze the difference between Interval-valued Fuzzy Sets (IVFSs) and Interval Type-2 Fuzzy Sets (IT2FSs), and to show that both are related to HFSs.

4.- To analyze the relationships between IVFSs, Atanassov Intuitionistic Fuzzy sets (AIFSs), Interval-Valued Atanassov Intuitionistic Fuzzy Sets (IVAIFSs), HFSs, SVFSs and T2FSs.

5.- To highlight some applications of each of the types of FSs.

Figure 1 presents a clear snapshot on the relationships 
TABLE I

HISTORY OF THE TYPES OF FUZZY SETS.

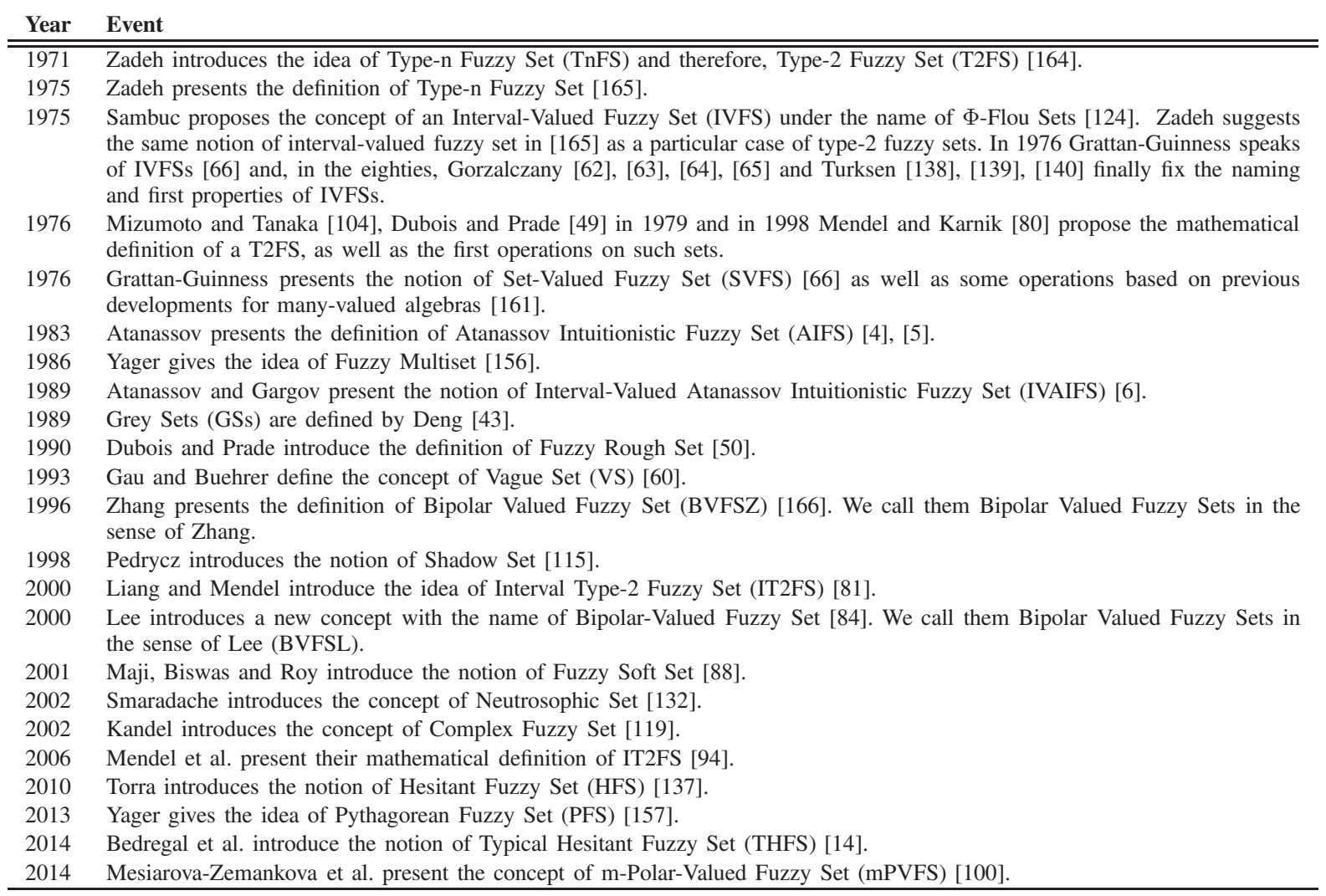

among the extensions. T2FSs encompass SVFSs and hence also hesitant sets, which include IVAIFSs. The latter contain IVFSs, which are mathematically identical to AIFSs. Finally, FSs are included in all of them.

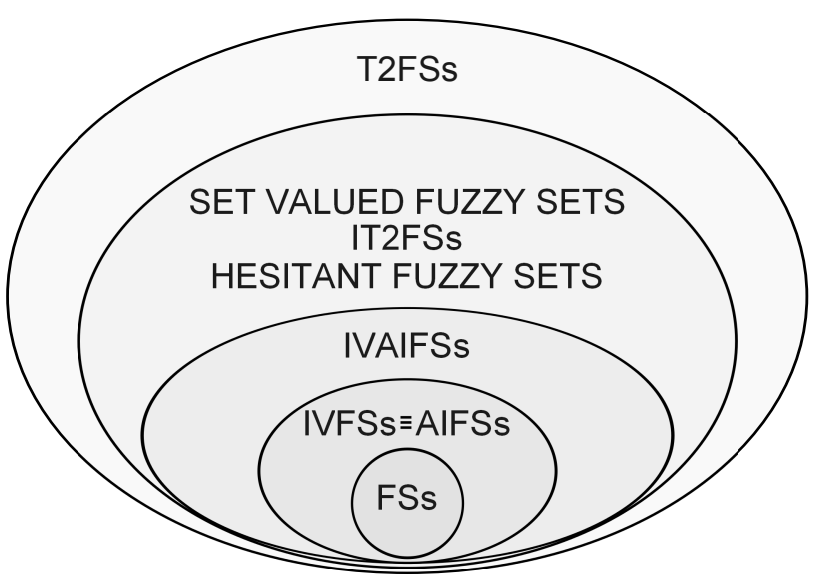

Fig. 1. Representation of the inclusion relationships between different types of fuzzy sets.

The paper starts by presenting the concepts of FSs and L-FSs (in Sections 2 and 3, respectively). In Section 4, we analyze T2FSs and their relationship with other types of FSs. Section 5 is devoted to Set-Valued Fuzzy Sets and Hesitant Fuzzy Sets. In Section 6, we study IVFSs and in Section 7 we analyze the case of IT2FSs. We then review in Section 8 AIFSs and the specific cases of Neutrosophic sets, BVFSZs and BVFSLs. We discuss in Sections 9, 10 and 11 IVAIFSs, Fuzzy Multisets and n-Dimensional Fuzzy Sets. In Section 12 we recall the definitions of Fuzzy Rough Sets, Fuzzy Soft Sets and Multi-Valued Fuzzy Sets. We finish with some conclusions and references.

\section{FUZZY SETS}

Łukasiewicz, together with Lesniewski, founded in the twenties of the XXth century a school of logic in Warsaw that became one of the most important mathematical teams in the world, and among whose members was Tarski. Eukasiewicz introduced the idea of distributing the truth values uniformly on the interval $[0,1]$ : if $n$ values are considered, then $0, \frac{1}{n-1}, \frac{2}{n-1}, \cdots, \frac{n-2}{n-1}, 1$ are the possible truth values; if there is an infinite number of truth values, one should take $\mathbb{Q} \cap[0,1]$. The negation is defined as $n(x)=1-x$, while $x \oplus y=\min (1, x+y)$ is also fixed.

In the line of Łukasiewicz's studies, Zadeh ([163]) introduced fuzzy sets in his 1965 work, Fuzzy Sets. His ideas on FSs were soon applied to different areas such as artificial intelligence, natural language, decision making, expert systems, neural networks, control theory, etc.

From now on, we denote by $X$ a non-empty universe, either finite or infinite.

Definition 2.1: A fuzzy set (or type-1 fuzzy set) $A$ on $X$ is a mapping $A: X \rightarrow[0,1]$.

The value $A(x)$ is referred to as the membership degree of the element $x$ to the fuzzy set $A$. 
An equivalent definition is given by $A=\left\{\left(x, \mu_{A}(x)\right) \mid x \in\right.$ $X\}$ with $\mu_{A}: X \rightarrow[0,1]$, explicitly providing the membership degree of each $x \in X$. Note that this definition attempts to identify the FS with the graph of the mapping $A$ given in Definition 2.1. Many other notations have been used for fuzzy sets in the literature. For example, in the early years of FS theory, a common way to describe a FS $A$ (see [104], for instance) on a finite universe $X$ was $A=\sum_{i=1}^{n} \mu_{A}\left(x_{i}\right) / x_{i}$, whereas the notation $A=\int_{X} \mu_{A}\left(x_{i}\right) / x_{i}$ was used for an infinite universe $X$. These definitions lead to important notational problems and misconceptions, since in fact no summation or integration is taking place.

We denote by $\mathrm{FS}(X)$ the class of FSs on the universe $X$ (note that, in fact, $\mathrm{FS}(X)=[0,1]^{X}$ ). A partial order relation $\leq_{\mathrm{F}}$ on $\mathrm{FS}(X)$ can be defined as follows. Given $A, B \in$ $\mathrm{FS}(X), A \leq_{\mathrm{F}} B$ if the inequality $A(x) \leq B(x)$ holds for every $x \in X$. Equivalently, we have the following important result.

Proposition 2.1: [163] $\left(\mathrm{FS}(X), \cup_{\mathrm{F}}, \cap_{\mathrm{F}}\right)$ is a complete lattice, where, for every $A, B \in \mathrm{FS}(X)$, union and intersection are defined, respectively, by

$$
\begin{aligned}
& A \cup_{\mathrm{F}} B(x)=\max (A(x), B(x)), \text { and } \\
& A \cap_{\mathrm{F}} B(x)=\min (A(x), B(x)) .
\end{aligned}
$$

It is important to recall that a lattice $L$ is a partially ordered set where, for each pair of elements, there exist a supremum and an infimum. If there exist a supremum and an infimum for every subset of $L$, then the lattice is called complete.

The first criticism to FS theory concerns the order relation $\leq_{\mathrm{F}}$. Despite Zadeh presented FSs in order to represent uncertainty, $\leq_{F}$ happens to be a crisp relationship. This fact has led Willmott [146], Bandler and Kohout [10] and others to consider the concept of inclusion measure. These measures have been widely used in fields such as mathematical morphology [42], or image processing [76].

When using the operations defined in Eqs. (1) and (2) together with the standard negation, $n(x)=1-x$ for all $x \in[0,1]$, neither the law of contradiction nor the law of the excluded middle hold. Nowadays, the operations in Eqs. (1) and (2) are expressed in terms of t-norms and t-conorms [28], [34], [58], [82].

Note that we can define a fuzzy set over the set of all fuzzy sets on a given universe $X$, leading to level 2 fuzzy sets [83]. Of course this can be generalized to level $k$ fuzzy sets [164].

\section{A GeneraliZATION: $L$-FUZZY SETS}

Goguen [61] realized that, other than its lattice structure, there was no relevant reason to use the interval $[0,1]$ in the definition of FSs. This observation led him to the introduction of the concept of an $L$-fuzzy set.

Definition 3.1: Let $L$ be a complete lattice. An $L$-fuzzy set $A$ on $X$ is a mapping $A: X \rightarrow L$.

Given a complete lattice $L$, the class of $L$-fuzzy sets on the universe $X$ is denoted by $L-F S(X)$. Note that, with this notation, if $L=[0,1]$ (and considering the $\max$ and $\min$ operations), then $\mathrm{FS}(X)$ is the same as $L-\mathrm{FS}(X)$. Again, $L-\mathrm{FS}(X)$ can be endowed with a partial order relation, which is induced by the lattice structure of $L$ as follows. Given $A, B \in L$-FS $(X), A \leq_{\mathrm{L}} B$ if the inequality $A(x) \leq_{\mathrm{L}} B(x)$ holds for every $x \in X$, where $\leq_{\mathrm{L}}$ denotes the order relation of the lattice $L$. Equivalently, we have the following result.

Proposition 3.1: [61] $\left(L-\mathrm{FS}(X), \cup_{\mathrm{LF}}, \cap_{\mathrm{LF}}\right)$ is a complete lattice, where, for every $A, B \in L$-FS $(X)$, union and intersection are defined, respectively, by:

$$
\begin{aligned}
& A \cup_{\mathrm{LF}} B(x)=A(x) \vee B(x), \text { and } \\
& A \cup_{\mathrm{LF}} B(x)=A(x) \wedge B(x),
\end{aligned}
$$

where $\vee$ is the greatest lower bound or meet operation and $\wedge$ is the least upper bound or join operation.

From Proposition 3.1 it is clear that FSs are a special case of $L$-fuzzy sets for which $L=[0,1]$ and the maximum and minimum take the role of the join and meet, respectively. The notion of $L$-FS allows some types of FSs to be emcompassed within a single theoretical framework.

\section{Type-2 FuZZY SETS}

\section{A. Origin of the Concept}

In 1971, and using the ideas given in [18], Zadeh settled in his work [164] that the problem of estimating the membership degrees of the elements to the fuzzy set is related to abstraction -a problem that plays a central role in pattern recognition. Therefore, the determination of the membership degree of each element to the set is the biggest problem for applying FS theory. Taking these considerations into account, the concept of type-2 fuzzy set was given as follows: A T2FS is a FS for which the membership degrees are expressed as FSs on [0,1].

Later, on December 11, 2008, Zadeh proposed the following definitions in the bisc-group mailing list:

Definition 4.1: Fuzzy logic is a precise system of reasoning, deduction and computation in which the objects of discourse and analysis are associated with information which is, or is allowed to be, imperfect.

Definition 4.2: Imperfect information is defined as information which in one or more respects is imprecise, uncertain, vague, incomplete, partially true or partially possible.

On the same date and place, Zadeh made the following remarks:

1.- In fuzzy logic, everything is or is allowed to be a matter of degree. Degrees are allowed to be fuzzy.

2.- Fuzzy logic is not a replacement for bivalent logic or bivalent-logic- based probability theory. Fuzzy logic adds to bivalent logic and bivalent-logic-based probability theory a wide range of concepts and techniques for dealing with imperfect information.

3.- Fuzzy logic is designed to address problems in reasoning, deduction and computation with imperfect information which are beyond the reach of traditional methods based on bivalent logic and bivalent logic-based probability theory.

4.- In fuzzy logic the writing instrument is a spray pen with precisely known adjustable spray pattern. In bivalent logic, the writing instrument is a ballpoint pen (see Fig. 2, which also appeared in the same place). 


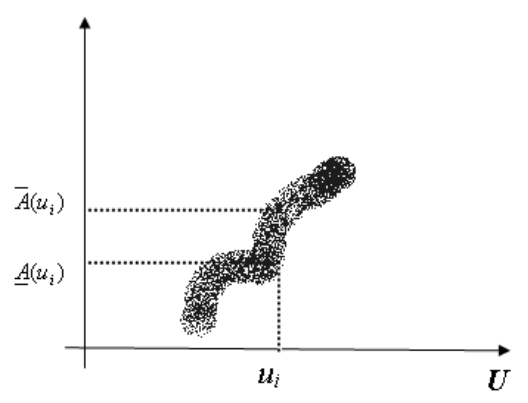

Fig. 2. Representation of the uncertainty in fuzzy logic, which according to Zadeh can be seen as a spray pen.

5.- The importance of fuzzy logic derives from the fact that in much of the real world, imperfect information is the norm rather than exception.

All of these considerations justify the use of FS theory whenever objects are linked to soft concepts, i.e. do not show clear boundaries. Nevertheless, the way to design membership functions might be non-evident, and faces a wide variety of difficulties. In such circumstances, it seems reasonable to make use of the so-called generalizations (types) of FSs, which might better accommodate the knowledge available in the context of the application. In fact, the introduction of many of such generalizations is directly associated to the need of building FSs that allow us to represent objects described through imperfect information, as well as to represent the lack of knowledge or uncertainty of the considered experts.

\section{B. Basic Definitions}

From the notion of T2FS given by Zadeh in [164], and the study made in [165], we have the following definition.

Definition 4.3: A T2FS $A$ on $X$ is a mapping $A: X \rightarrow$ $\operatorname{FS}([0,1])$.

From Definition 4.3 it can be seen that, mathematically, a T2FS is a mapping $A: X \rightarrow[0,1]^{[0,1]}$. We denote by $\mathrm{T} 2 \mathrm{FS}(X)$ the class of T2FSs on the universe $X$.

Note that any $A \in \mathrm{FS}(X)$ can also be seen as a T2FS for which the membership degree is given by a singleton on $[0,1]$, that is:

$$
S(t)= \begin{cases}1 & \text { if } t=A(x) \\ 0 & \text { otherwise. }\end{cases}
$$

Elaborating on Zadeh's definitions for union and intersection of FSs, Mizumoto and Tanaka [104] in 1976 and Dubois and Prade [49] in 1979, proposed the following definition of union and intersection for T2FSs.

Definition 4.4: For every $A, B \in \mathrm{T} 2 \mathrm{FS}(X)$,

$$
\begin{aligned}
& A \cup_{\mathrm{T} 2 \mathrm{~F}} B(x)=A(x) \cup_{\mathrm{F}} B(x), \text { and } \\
& A \cap_{\mathrm{T} 2 \mathrm{~F}} B(x)=A(x) \cap_{\mathrm{F}} B(x) .
\end{aligned}
$$

Proposition 4.1: (T2FS $\left.(X), \cup_{\mathrm{T} 2 \mathrm{~F}}, \cap_{\mathrm{T} 2 \mathrm{~F}}\right)$ is a complete lattice.

With the union and intersection given in Definition 4.4 the classical definitions of union and intersection $\cup_{F}$ and $\cap_{F}$ given by Zadeh for FSs [48] are not recovered. Consider a finite universe $X=\left\{x_{1}, x_{2}, x_{3}\right\}$, and consider the following FSs on $X$ (see [22]):

$$
\begin{aligned}
& A=\left\{\left(x_{1}, \frac{1}{2}\right),\left(x_{2}, \frac{1}{3}\right),\left(x_{3}, 1\right)\right\}, \text { and } \\
& B=\left\{\left(x_{1}, \frac{1}{4}\right),\left(x_{2}, \frac{1}{2}\right),\left(x_{3}, \frac{1}{7}\right)\right\} .
\end{aligned}
$$

We have that, for instance, $A \cup_{\mathrm{F}} B=$ $\left\{\left(x_{1}, \frac{1}{2}\right),\left(x_{2}, \frac{1}{2}\right),\left(x_{3}, 1\right)\right\}$. Alternatively, let $A_{T 2}$ and $B_{T 2}$ be analogous T2FSs, i.e.:

$$
\begin{aligned}
& A_{T 2}\left(x_{1}\right)(t)= \begin{cases}1 & \text { if } t=\frac{1}{2} \\
0 & \text { otherwise }\end{cases} \\
& A_{T 2}\left(x_{2}\right)(t)= \begin{cases}1 & \text { if } t=\frac{1}{3} \\
0 & \text { otherwise }\end{cases} \\
& A_{T 2}\left(x_{3}\right)(t)= \begin{cases}1 & \text { if } t=1 \\
0 & \text { otherwise }\end{cases}
\end{aligned}
$$

and

$$
\begin{aligned}
& B_{T 2}\left(x_{1}\right)(t)= \begin{cases}1 & \text { if } t=\frac{1}{4} \\
0 & \text { otherwise }\end{cases} \\
& B_{T 2}\left(x_{2}\right)(t)= \begin{cases}1 & \text { if } t=\frac{1}{2} \\
0 & \text { otherwise }\end{cases} \\
& B_{T 2}\left(x_{3}\right)(t)= \begin{cases}1 & \text { if } t=\frac{1}{7} \\
0 & \text { otherwise. }\end{cases}
\end{aligned}
$$

Then we have that

$$
\begin{aligned}
& A_{T 2} \cup_{\mathrm{T} 2 \mathrm{~F}} B_{T 2}\left(x_{1}\right)(t)= \begin{cases}1 & \text { if } t=\frac{1}{4} \text { or } t=\frac{1}{2} \\
0 & \text { otherwise }\end{cases} \\
& A_{T 2} \cup_{\mathrm{T} 2 \mathrm{~F}} B_{T 2}\left(x_{2}\right)(t)= \begin{cases}1 & \text { if } t=\frac{1}{2} \text { or } t=\frac{1}{3} \\
0 & \text { otherwise }\end{cases} \\
& A_{T 2} \cup_{\mathrm{T} 2 \mathrm{~F}} B_{T 2}\left(x_{3}\right)(t)= \begin{cases}1 & \text { if } t=\frac{1}{7} \text { or } t=1 \\
0 & \text { otherwise }\end{cases}
\end{aligned}
$$

which does not coincide with our previous result. Moreover, observe that a T2FS is recovered, instead of a FS.

Alternative definitions of union and intersection have been provided for T2FSs extending Zadeh's union and intersection [70], [73]. For example, the operators

$$
\begin{aligned}
& \left(A \sqcup_{\mathrm{T} 2 \mathrm{~F}} B\right)(x)=\sup \{\min (A(y), B(z)) \mid \max (y, z)=x\} \\
& \left(A \sqcap_{\mathrm{T} 2 \mathrm{~F}} B\right)(x)=\sup \{\min (A(y), B(z)) \mid \min (y, z)=x\}
\end{aligned}
$$

are based on Zadeh's extension principle.

Remark 1: Note that $\left(\mathrm{T} 2 \mathrm{FS}(X), \sqcup_{\mathrm{T} 2 \mathrm{~F}}, \sqcap_{\mathrm{T} 2 \mathrm{~F}}\right)$ becomes a basic algebra, but not a lattice, since the absorption law does not hold (see [71], [72], [73]).

From the ideas given by Karnik and Mendel [80] in 1998, Mendel and John [93] provide in 2002 the following definition:

Definition 4.5: A T2FS $A$ is characterized by a type-2 membership function $\mu_{A}(x, u)$, where $x \in X$ and $u \in J_{x} \subseteq$ $[0,1]$, i.e.,

$$
A=\left\{\left(x, \mu_{A}(x, u)\right) \mid x \in X, u \in J_{x} \subseteq[0,1]\right\},
$$


in which $0 \leq \mu_{A}(x, u) \leq 1$. $A$ can also be expressed as

$$
A=\int_{x \in X} \int_{u \in J_{x}} \mu_{A}(x, u) /(x, u), J_{x} \subseteq[0,1],
$$

where $\iint$ denotes union over all admissible $x$ and $u$.

For discrete universes, $\int$ is replaced by $\sum$. From a mathematical point of view, however, this definition leads to several problems. Apart from the confusion that may arise from the use of integral (or summation) symbols when no such operations are actually carried out, the use of a subset $J_{x}$ may lead to ambiguities and even to conceptual difficulties, as we see in the case of Interval T2FSs. In any case, the equivalence between Definition 4.3 and the one by Mendel and John is straightforward. Just recall that, given $A \in \mathrm{FS}([0,1])$, its support is defined as

$$
\operatorname{supp}(A)=\{t \in[0,1] \mid A(t)>0\} .
$$

Following the notation by Mendel and John, $J_{x}=\operatorname{supp}(A(x))$ and $\mu_{A}(x, \cdot)=A(x)$.

In Fig. 3, we display the most relevant contributions to the mathematical formalization of the notion of a T2FS.

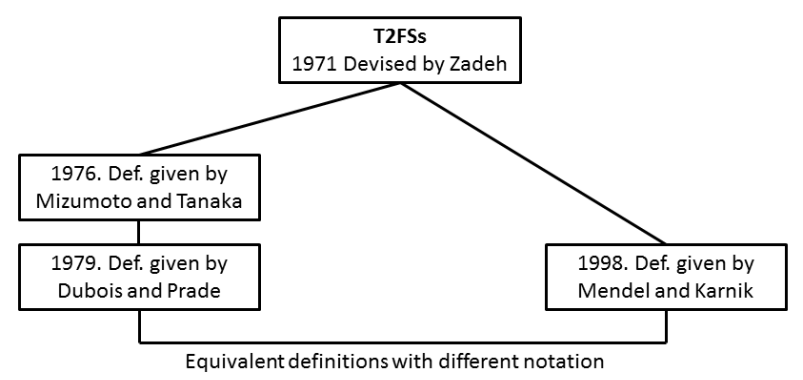

Fig. 3. Schematic representation of the history of the mathematical formalization of T2FS.

\section{Some Considerations about T2FSs}

Next, some important aspects about T2FSs are considered:

1.- Notation. Mizumoto and Tanaka in 1976 [104] and Mendel and John in 2002 [93] used the following notation:

$$
\int_{x \in X} \int_{t \in J_{x}} A(x, t) /(x, t) \quad J_{x} \subset[0,1],
$$

where $J_{x}$ is the primary membership of $x \in X$ and, for each fixed $x=x_{0}$, the FS $\int_{t \in J_{x_{0}}} A\left(x_{0}, t\right) / t$ is the secondary membership of $x_{0}$.

We propose a clarifying notation as follows: observe that a T2FS assigns to an element in the universe $X$ a mapping $A(x):[0,1] \rightarrow[0,1]$. It is quite usual to represent FSs (or type-1 fuzzy sets) by a mapping $A$

$$
\{(x, A(x)) \mid x \in X\} .
$$

In this type- 1 case, $A(x)$ is a real number in $[0,1]$ for every $x \in X$. In the case of type-2 fuzzy sets, we have that $A(x)$ is a mapping (a type-1 fuzzy set) instead of a real number, i.e.,

$$
\begin{aligned}
A(x):[0,1] & \rightarrow[0,1] \\
t & \mapsto A(x)(t) .
\end{aligned}
$$

Taking these considerations into account, Walker and Walker in [70] suggested the following notation for a T2FSs:

$$
A=\{(x,(t, A(x)(t))) \mid x \in X, t \in[0,1]\} .
$$

An easier notation, following the ideas in Aisbett, Rickard and Morgenthaler in [1], is the following one.

Definition 4.6: Let $A: X \rightarrow \mathrm{FS}([0,1])$ be a type-2 fuzzy set. Then $A$ is denoted as

$$
\{(x, A(x, t)) \mid x \in X, t \in[0,1]\},
$$

where $A(x, \cdot):[0,1] \rightarrow[0,1]$ is defined as $A(x, t)=$ $A(x)(t)$.

2.- Computational efficiency. T2FSs lead to an increasing computational complexity compared to T1FSs. However, there have been significant efforts in speeding up the computational analysis and handling of T2FSs.

3.- Type-n Fuzzy Sets (TnFSs). Type-3 fuzzy sets can also be defined as FSs for which the membership degree of each element is given by a T2FS [83]. Moreover, it is possible to define recursively type-n fuzzy sets as fuzzy sets whose membership values are type-(n-1) fuzzy sets. The computational efficiency of these sets decreases as the complexity level increases. From a theoretical point of view, it is necessary to carry out a complete analysis of type-n fuzzy set structures and operations. But up to now, no applications have been developed using TnFSs.

4.- Applicability. Various applications have employed type2 fuzzy sets, including the work of Mendel et al. on computing with words and perceptual computing [95], [96], [98], [99], by Hagras [68], [69], by Castillo in control [35], and by Xia et al. in networks ([150]).

\section{Set-Valued Fuzzy Sets and Hesitant Fuzzy Sets}

\section{A. Set-Valued Fuzzy Sets}

In 1976, Grattan-Guinness [66] defined SVFSs as FS for which membership degrees are expressed as subsets $[0,1]$. Formally, we have the following definition.

Definition 5.1: A SVFS $A$ on $X$ is a mapping $A: X \rightarrow$ $2^{[0,1]} \backslash\{\emptyset\}$.

Here $2^{[0,1]}$ is the power set of $[0,1]$, that is, the set of all subsets of $[0,1]$.

We denote by $\operatorname{SVFS}(X)$ the class of all SVFSs on $X$. A SVFS $A$ can be seen as a T2FS $B_{A}$ by defining, for each $x \in X$, the FS $B_{A}(x):[0,1] \rightarrow[0,1]$ as follows

$$
B_{A}(x)(u)= \begin{cases}1 & \text { if } u \in A(x) \\ 0 & \text { otherwise }\end{cases}
$$

As the set $2^{[0,1]}$, with the classical definitions of union and intersection between (crisp) sets, is a bounded lattice, we have the following result. 
Proposition 5.1: $\left(\operatorname{SVFS}(X), \cup_{\mathrm{SVF}}, \cap_{\mathrm{SVF}}\right)$ is a complete lattice, where, for every $A, B \in \operatorname{SVFS}(X)$,

$$
\begin{aligned}
& A \cup_{\mathrm{SVF}} B(x)=A(x) \cup B(x), \\
& A \cap_{\mathrm{SVF}} B(x)=A(x) \cap B(x) .
\end{aligned}
$$

However, as in the case of T2FSs, with $\cup_{\text {SVF }}$ and $\cap_{\text {SVF }}$ the union and intersection of FSs, as defined by Zadeh, are not recovered. Grattan-Guinness did not consider this problem in his work, but a possible solution was provided by Torra [137], as explained in the following section.

Remark 2: An interesting open problem is to provide a lattice structure on $\operatorname{SVFS}(X)$ in such a way that Zadeh's union and intersection are preserved when restricted to FSs.

\section{B. Hesitant Fuzzy Sets}

In [137], Torra proposed to express membership degrees as subsets of $[0,1]$, giving birth to the so-called Hesitant Fuzzy Sets (HFSs), which he defined as "a function that when applied to $X$ returns a subset of [0,1]" [137]. Clearly, this definition turns out to be exactly the same as SVFSs. However, in [137], and contrary to what Grattan-Guinness did, Torra proposed a definition of union and intersection for SVFSs that extends those by Zadeh. Given $A, B \in \operatorname{SVFS}(X)$, Torra defined:

$$
\begin{aligned}
& \quad A \cup_{\mathrm{HF}} B(x)= \\
& \{t \in A(x) \cup B(x) \mid t \geq \max (\inf A(x), \inf B(x))\}, \text { and } \\
& \quad A \cap_{\mathrm{HF}} B(x)= \\
& \{t \in A(x) \cup B(x) \mid t \leq \min (\sup A(x), \sup B(x))\} .
\end{aligned}
$$

\section{Some Considerations about HFSs}

Next, some important aspects about HFSs are considered.

1.- Unfortunately, $\left(\operatorname{HFS}(X), \cup_{\mathrm{HF}}, \cap_{\mathrm{HF}}\right)$ is not a lattice. For example, let $X=\{x\}$. Consider the SVFSs $A$ and $B$ with $A(x)=\{0.3,0.5,0.6\}$ and $B(x)=\{0.4,0.5,0.7,0.8\}$, then $A \cap_{\mathrm{HF}} B(x)=\{0.3,0.4,0.5,0.6\}$ and therefore $A \cup_{\mathrm{HF}}\left(A \cap_{\mathrm{HF}} B\right)(x)=\{0.3,0.4,0.5,0.6\}$ which is different from $A(x)$. Consequently $A \cup_{\mathrm{HF}}\left(A \cap_{\mathrm{HF}} B\right) \neq A$, and the law of absorption does not hold.

2.- Recently, HFSs have been used in group decision making problems [123], and extended to Computing with Words [121], [122].

\section{Typical Hesitant Fuzzy Sets}

When the membership degree of each of the elements is given by a finite and non-empty subset of $[0,1]$, HFSs are called Typical Hesitant Fuzzy Sets (THFSs) [14], [16]. Most of the works applying HFSs actually make use of THFSs [123].

\section{INTERVAL-VALUED FUZZY SETS}

\section{A. Basic Definitions}

In 1975 Sambuc [124] presented the concept of IntervalValued Fuzzy Set (IVFS) with the name of $\Phi$-fuzzy set. Zadeh suggested the same notion of interval-valued fuzzy set in page 242 of [165] as a particular case of type-2 fuzzy sets.
That same year, Jahn [78] wrote about these sets. One year later, Grattan-Guinness [66] established a definition of an IV membership function. In the same decade IVFSs appeared in the literature in various guises and it was not until the 1980s, with the work of Gorzalczany and Türksen [56], [62], [64], [65], [138], [140], [141] that the importance of these sets, as well as their name, was definitely established.

Let us denote by $L([0,1])$ the set of all closed subintervals of $[0,1]$, i.e.,

$$
L([0,1])=\left\{[\underline{x}, \bar{x}] \mid(\underline{x}, \bar{x}) \in[0,1]^{2} \text { and } \underline{x} \leq \bar{x}\right\} .
$$

Definition 6.1: [124] An IVFS $A$ on $X$ is a mapping $A$ : $X \rightarrow L([0,1])$.

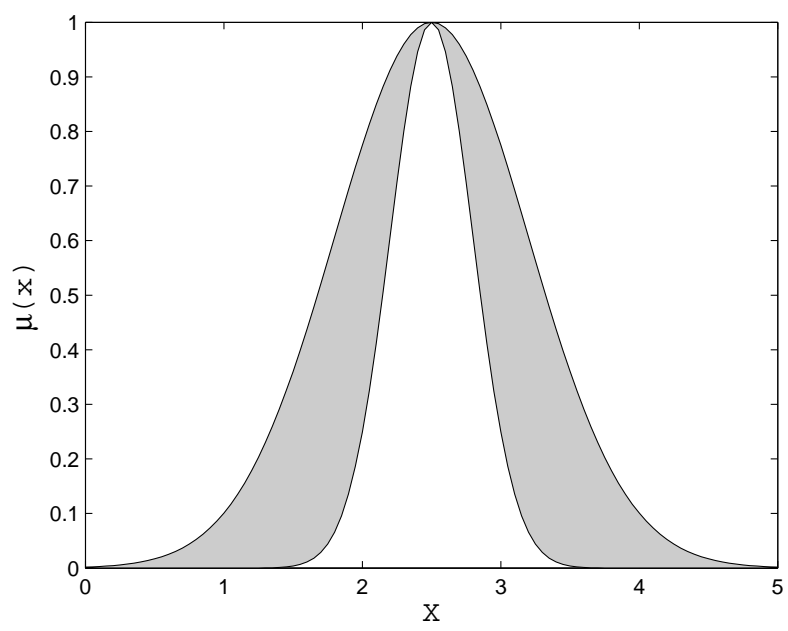

Fig. 4. Example of an interval-valued fuzzy set.

The membership degree of $x \in X$ to $A$ is given by $A(x)=$ $[\underline{A}(x), \bar{A}(x)] \in L([0,1])$, where the mappings $\underline{A}: X \rightarrow[0,1]$ and $\bar{A}: X \rightarrow[0,1]$ correspond to the lower and the upper bounds of the membership interval $A(x)$, respectively. An example of an IVFS can be seen in Fig. 4. We denote by $\operatorname{IVFS}(X)$ the class of IVFSs on the universe $X$.

Obviously, if $\underline{A}(x)=\bar{A}(x)$ for every $x \in X$, the considered set is a FS. So, FSs are particular cases of interval-valued fuzzy sets.

In 1989, Deng [43] presented the concept of grey sets. Dubois proved that these sets coincide with IVFSs [51].

Besides, the so-called shadowed sets (SSs) were suggested by Pedrycz [115] and further studied by Pedrycz and Vukovich [116], [117]. Given a $A \in \mathrm{FS}(X)$, a shadowed set $B$ induced by a $A$ is an IVFS on $X$ such that the membership degree of an element $x \in X$ is either $[0,0]$, [1, 1$]$ or $[0,1]$; i.e. $B$ is a mapping $B: U \rightarrow\{0,1,[0,1]\}$, where $0,1,[0,1]$ denote complete exclusion from $B$, complete inclusion in $B$ and complete ignorance, respectively. It is easy to see that these sets are a particular case of IVFSs.

Regarding the lattice structure of IVFSs, we can state the following.

Proposition 6.1: Given a universe $X$, $\left(\operatorname{IVFS}(X), \cup_{\mathrm{IV}}, \cap_{\mathrm{IV}}\right)$, with the operations defined, for 
$A, B \in \operatorname{IVFS}(X)$ and for every $x \in X$, by:

$$
\begin{aligned}
& A \cup_{\mathrm{IV}} B(x)=[\max (\underline{A}(x), \underline{B}(x)), \max (\bar{A}(x), \bar{B}(x))], \\
& A \cap_{\mathrm{IV}} B(x)=[\min (\underline{A}(x), \underline{B}(x)), \min (\bar{A}(x), \bar{B}(x))],
\end{aligned}
$$

is a bounded lattice.

Note that $\left(\mathrm{L}([0,1]), \cup_{\mathrm{IV}}, \cap_{\mathrm{IV}}\right)$ is a bounded lattice, where, for every $[a, b],[c, d] \in \mathrm{L}([0,1])$, we have:

$$
\begin{aligned}
& {[a, b] \cup_{\mathrm{IV}}[c, d]=[\max (a, c), \max (b, d)],} \\
& {[a, b] \cap_{\mathrm{IV}}[c, d]=[\min (a, c), \min (b, d)] .}
\end{aligned}
$$

From Proposition 6.1 it can be stated that IVFSs are a particular case of $L$-fuzzy sets.

\section{B. Different Interpretations of IVFSs}

Two different semantics can be considered for IVFSs [108]:

1) The membership degree of an element to the set corresponds to a value in the considered membership interval. We can not say in a precise way what that value is, so we just provide bounds for it.

2) The membership degree of each element is the whole closed subinterval provided as membership, understood as an element in the lattice of closed subintervals of the unit interval. From a mathematical point of view, this interpretation is very appealing, but, in our opinion, it is not easy to see what it implies in the applied field. Moreover, in this case, we find the following paradox [25].

For FSs and with the standard negation it holds that $\min (A(x), 1-A(x)) \leq 0.5$ for all $x \in X$. But for IVFSs, if we also use the standard negation $N(A(x))=$ $[1-\bar{A}(x), 1-\underline{A}(x)]$, there is no equivalent bound for

$$
\min ([\underline{A}(x), \bar{A}(x)],[1-\bar{A}(x), 1-\underline{A}(x)]) .
$$

Remark 3: Some authors consider that, when working with IVFSs, the fact that an analogon of the inequality $\min (A(x), 1-A(x)) \leq 0.5$ does not hold is a problem for the use of IVFSs. However, from our point of view, such objection is similar to the criticism to Zadeh's theory when he showed that fuzzy logic does not satisfy the law of contradiction and the law of excluded middle. So we consider that the lack of such an inequality does not make invalid the application and study of IVFSs.

\section{Relationships Between IVFSs, SVFSs, HFSs and T2FSs}

There exist certain relationships between the extensions reviewed so far.

Proposition 6.2: [26] IVFSs are a particular case of SVFSs (and hence of HFSs and therefore also of T2FSs).

In particular, if $A$ is an IVFS, a SVFS $h_{A}$ is recovered by

$$
h_{A}(x)=[\underline{A}(x), \bar{A}(x)]=A(x)
$$

for every $x \in X$. Assuming that $A, B \in \operatorname{IVFS}(X)$, they can be treated as SVFSs. Then, from Eq. (12)

$$
h_{A \cup_{\mathrm{HF}} B}(x)=[\max (\underline{A}(x), \underline{B}(x)), \max (\bar{A}(x), \bar{B}(x))]
$$

and

$$
h_{A \cap_{\mathrm{HF}} B}(x)=[\min (\underline{A}(x), \underline{B}(x)), \min (\bar{A}(x), \bar{B}(x))] .
$$

This leads to the following result.

Proposition 6.3: Union and intersection of IVFSs considered as HFSs are the same as union and intersection of IVFSs themselves.

In 1995, Klir and Yuan showed [83] that, from an IVFS, a T2FS can be built as in Figure 5. In this figure, the membership interval for each point $u_{i}$ in the referential set is defined by the lower and the upper bound of the shadowed area at that point. To build the fuzzy set that defines the membership of $u_{i}$ to the corresponding type-2 FS, the membership for each point (of $[0,1]$ ) inside that shadowed area is made equal to one. Finally, in order to get a continuous membership function, we build a trapezoidal membership function by slightly displacing the lower and the upper bounds.

Later, in 2007 Deschrijver and Kerre [45], [46] and Mendel [90], showed that IVFSs are particular cases of T2FSs.

\section{Some Considerations about IVFSs}

Next, some important aspects of IVFSs are considered:

1.- Measures yielding intervals. From the definition of IVFSs, and following Gorzalczany [62], the compatibility degree between two IVFSs can be defined as an element in $L([0,1])$. Other information measures (interval-valued entropy, interval-valued similarity, etc.) [15], [21], [27], [79], [111], [120], [134] should also be given by an interval. However, most of the works proposing measures, produce scalar measurements instead of interval ones. It is clear, however, that from a theoretical point of view, two different types of information measures should be discriminated: those that yield scalars and those that yield interval-valued measurements. Obviously, problems arise in the interpretation of both types of measures. Moreover, if the result of the measure is an interval, its length reflects the lack of knowledge [32] linked to the considered measurement.

2.- Computational cost. Due to the current developments in hardware and computer machinery, the computational load of working with IVFSs is not significantly greater than that of working with type-1 fuzzy sets [147].

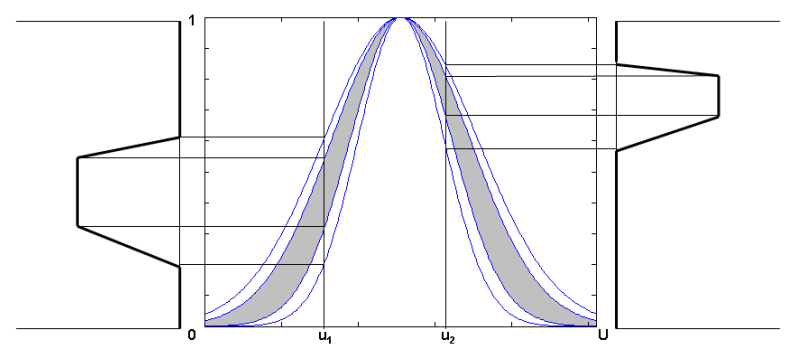

Fig. 5. Construction of T2FSs from IVFSs 
3.- Linear orders. Often non-linear orders are used to sort intervals, which leads to undesired problems in applications where sorting takes a key role, e.g. decision making. In [29] it is shown that the order that must be used for a given application depends on the application itself. Namely, the same order should not be used for all possible applications. In particular, in those applications where a linear order is needed in order to compare inputs (e.g., in decision making problems, for instance, see [29]), experts may not have enough information to choose or produce such total order. This fact can lead to inconsistent results, since the choice of the order influences strongly the final outcome. In [33] some methods to build linear orders among intervals, for those applications where such orders are needed, are explained.

4.- Applications. There exist a variety of works in the literature in which the use of IVFSs allows for an improvement of the results obtained with T1FSs. Moreover, and from a cognitive point of view, it comes out that, for these applications, the definition of parameters is not more complicated than the definition of the parameters for their fuzzy counterparts [125]. For instance: In classification problems [125], [126], [127], [128], [130]. The experimental results presented in [128] show that the approach using IVFSs (named IVTURS) improves the results of two state-of-the-art fuzzy classifiers like FARCHD [2] and FURIA [77]. Image processing. IVFSs can be used for representing those areas of an image where experts have problems to build the fuzzy membership degrees. In this case, The use of IVFSs leads to improved segmentation or detection of features [11], [30], [31], [32], [59], [109], [136], [162]. For some decision making problems, the use of IVFSs allows to choose a solution when FSs fail to do so [36]. Finally, IVFSs have also been used successfully in web problems [86], pattern recognition [39], medicine [40], etc. see also [97], [149].

\section{INTERVAL TYPE-2 FUZZY SETS ARE AN UNINTENDED GENERALIZATION OF IVFSS}

From the notion given by Karnik and Mendel in 1998 of T2FSs and Definition 4.5, Interval Type 2 Fuzzy Sets were defined in 2006 (see [94]) as follows.

Definition 7.1: When all $\mu_{A}(x, u)=1$, then $A$ is an IT2FS. Hence, according to [94], an IT2FS corresponds to

$$
A(x)=\left\{(u, 1) \mid u \in J_{x} \subseteq[0,1]\right\}
$$

for every $x \in X$.

It has been proven in [26] that IT2FSs represent a generalization of the concept of IVFSs and that both concepts are not equivalent.

Note that in the definition given in [94] (Eq. (5)) $J_{x} \subseteq[0,1]$ is used, so this is also the case for Definition 7.1 obtained from Eq. (5). However, it is well known that with such mathematical expression $J_{x}$ may be any subset of $[0,1]$, not just a closed subinterval. Hence, this is similar to the SVFS definition given by Grattan-Guinness in 1976 in [66]. Hence, for any IT2FS $A$, it is enough to consider the SVFS $h_{A}$ given by $h_{A}(x)=J_{x}$ for every $x \in X$.
In the literature, there has been a confusion about IT2FSs and IVFSs where it was mentioned in some work that both concepts are the same [90].

\section{Atanasssov Intuitionistic FuZzy Sets}

\section{A. Basic Definitions}

In 1983, Atanassov presented [4] his definition of AIFS. This work was written in Bulgarian, while in 1986 he presented these ideas in English [5].

Definition 8.1: An AIFS $A$ on $X$ is a mapping

$$
A: X \rightarrow D([0,1])=\left\{(x, y) \in[0,1]^{2} \mid x+y \leq 1\right\} .
$$

$A(x)=\left(\mu_{A}(x), \nu_{A}(x)\right)$ for all $x \in X$, where $\mu_{A}(x)$ is the membership degree of the element $x$ to $A$ and $\nu_{A}(x)$ is the non-membership degree. Both values should satisfy the restriction

$$
0 \leq \mu_{A}(x)+\nu_{A}(x) \leq 1
$$

Atanassov also introduced two definitions for these sets:

1.- The complement of $A(x)=\left(\mu_{A}(x), \nu_{A}(x)\right)$ is $A_{c}=$ $\left(\nu_{A}(x), \mu_{A}(x)\right)$;

2.- For each $x \in X$, the intuitionistic or hesitance index in the considered set $A$ is given by:

$$
\pi_{A}(x)=1-\mu_{A}(x)-\nu_{A}(x) .
$$

Note that $\pi_{A}(x)$ is a measure of the hesitation of the expert to assign a numerical value to $\mu_{A}(x)$ and $\nu_{A}(x)$. For this reason, we consider AIFSs to be an extension of FSs in the sense given by Zadeh in 1971.

In $1993 \mathrm{Gau}$ and Buehrer [60] introduced the concept of Vague Set and later, in 1994, it was shown to be the same as AIFS [23].

B. Relaxing the Restriction on the Membership Degrees: Neutrosophic Sets, Bipolar Valued Fuzzy Sets, Pythagorean Fuzzy Sets

If, for each $x \in X$, we take $\mu_{A}(x)+\nu_{A}(x)=1$, then the considered set $A$ is a FS in Zadeh's sense. So, FSs are a particular case of those defined by Atanassov.

From Eq. (18), the following inequality follows:

$$
\min \left(\mu_{A}(x), \nu_{A}(x)\right) \leq 0.5 .
$$

If, in Definition 8.1, the restriction given in Eq. (18) is relaxed, we obtain Neutrosophic sets (NSs) as defined by Smaradache in 2002 [132], and Pythagorean fuzzy sets (PFSs), defined by Yager in 2013 [157].

Definition 8.2: A NS $A$ on $X$ is a mapping $A: X \rightarrow$ $[0,1]^{2}$.

Definition 8.3: A PFS $A$ on $X$ is a mapping

$$
A: X \rightarrow\left\{(x, y) \in[0,1]^{2} \mid x^{2}+y^{2} \leq 1\right\} .
$$

Yager studied the negation operation and its relationship to the Pythagorean theorem. He compared Pythagorean fuzzy sets with AIFSs. The former are used currently in Decision Making [158]. In any case, the following result is straight.

Corollary 8.1: AIFSs are PFSs. 
Allowing to consider a negative value for $\nu_{A}(x)$, we find the bipolar valued fuzzy sets, presented by Zheng in 1996 [166].

Definition 8.4: A bipolar valued fuzzy set in the sense of Zhang (BVFSZ) $A$ on $X$ is a mapping $A: X \rightarrow[0,1] \times$ $[-1,0]$.

It is usual to denote $A(x)=\left(\varphi^{+}(x), \varphi^{-}(x)\right)$ with

$$
\begin{aligned}
& \varphi^{+}: X \rightarrow[0,1], \\
& \varphi^{-}: X \rightarrow[-1,0] .
\end{aligned}
$$

Obviously, $\varphi^{+}(x)+\varphi^{-}(x) \in[-1,1]$. In these sets, $\varphi^{-}(x)$ captures how much the environment of the problem opposes to the fulfillment of $\varphi^{+}(x)$. Nowadays, several works exist about these sets [167], [168].

Besides, in 2000, Lee [84] uses the name of bipolar-valued fuzzy sets to represent the following sets.

Definition 8.5: A bipolar-valued fuzzy set in the sense of Lee (BVFSL) on $X$ is a mapping

$$
A: X \rightarrow[-1,1] \text {. }
$$

Note that a BVFSL is mathematically very similar to a FS, since the only change is that the range of the membership function $A$ is $[-1,1]$ instead of $[0,1]$. However, the semantics are different. In fact, the name of bipolar-valued fuzzy set comes from the fact that a bipolar scale is considered, with negative valued considered to be opposite from positive ones. Observe that also Zhang's BVFS can be seen as bipolar, although involving two poles in two different scales. Moreover, BVFSLs are just a particular case of BVFSZs, since given $A \in \operatorname{BVFSL}(X)$ :

$$
A=\{(x, A(x)) \mid x \in X\},
$$

it can be seen as identical to the BVFSZ

$$
\hat{A}=\{(x, \max (A(x), 0), \min (A(x,), 0)) \mid x \in X\} .
$$

Note the pole -1 in Lee's sets corresponds to the membership degree $(0,-1)$, whereas the pole 1 corresponds to $(1,0)$.

AIFSs are also a particular case of bipolar-valued fuzzy set. Even more, and regarding Zhang's approach, if we have an Atanassov intuitionistic pair $(\mu, \nu)[151]$, with $\mu, \nu \in[0,1]$ and $\mu+\nu \leq 1$, since we can consider the linear transformation $F:[0,1] \rightarrow[-1,0]$ given by $F(t)=t-1$, we see that the pair $(\mu, \nu)$ becomes a new pair $(p, q) \in[0,1] \times[-1,0]$ with $p+q=\mu+\nu-1 \leq 0$.

\section{Relationships Between AIFSs, IVFSs, SVFSs and HFSs}

In 1989 Atanassov and Gargov [6], and later Deschrijver and Kerre [45], proved that from an IVFS we can build an AIFS and vice versa.

Theorem 8.2: The mapping

$$
\begin{aligned}
\Phi: \operatorname{IVFS}(X) & \rightarrow \operatorname{AIFS}(X) \\
A & \mapsto A^{\prime}
\end{aligned}
$$

where $A^{\prime}(x)=\left(\mu_{A}(x), \nu_{A}(x)\right)=(\underline{A}(x), 1-\bar{A}(x))$, is a bijection.

Theorem 8.2 shows that IVFSs and AIFSs are identical from a mathematical point of view. With this identity in mind, we have the following corollary of Proposition 6.2.
Corollary 8.3: AIFSs are a particular case of HFSs and hence of SVFSs. Moreover, the union and intersection of AIFSs are preserved if they are considered as HFSs.

Although IVFSs and AIFSs are mathematically identical, from a conceptual point of view, they are completely different. As pointed out in [108], the absence of a structural component in their description might explain this result:

(a) The representation of the membership of an element to a set using an interval means that the expert doubts about the exact value of such membership, so such an expert provides two bounds, and we never consider the representation of the non-membership to a set.

(b) By means of the intuitionistic index, we represent the hesitance of the expert in simultaneously building the membership and the non-membership degrees.

From an applied point of view, the difference between both concepts has also been clearly shown by Ye in [159]. On page 204 of this work, Ye adapted an example by Herrera and Herrera-Viedma [74]. Ye's example runs as follows: $n$ experts are asked about a money investment in four different companies. Ye considered that the membership to the sets that represent each of the companies is given by the number of experts that would invest their money in that company (normalized by $n$ ), and the non-membership is given by the number of experts that would not do so. Clearly, the intuitionistic index corresponds to the experts that neither provide a positive nor a negative answer about investing in that company. In this way, Ye showed that:

1.- The results obtained with this representation are closer to the decision of investors than those obtained in [74] using Zadeh's FSs.

2.- In the considered problem, the interval interpretation does not make much sense besides its use as a mathematical tool.

\section{Some Considerations about AIFSS}

Next, some important aspects about AIFSs are considered.

1.- Measures yielding pairs. Each membership degree in a AIFSs is bivalued. For this reason, we consider that information measures such as entropy [21], [134] or similarity [9], [133] should also yield bivalued measurements. As happened with IVFSs, a discrimination should be made between measures yielding scalar or bivalued measurements. This fact is discussed in [17], [110], where the two interpretations of entropy [21], [134] are jointly used to represent the uncertainty linked to an AIFS. We think that it is necessary to carry out a conceptual revision of the definitions of similarity, dissimilarity, entropy, comparability, etc., given for these sets. This becomes more relevant given the light computational overhead in working with two numbers instead of with a single one.

2.- Linear orders. In many applications it is problematic to choose the most appropriate linear order associated to that application [33], [151]. We should remark that the chosen order directly influences the final outcome, so it is necessary to study the conditions that determine the choice of one order or another [33]. 
3.- Terminological problem with the name intuitionistic. It is well known that the term intuitionistic was used in 1907 by Brouwer, in 1930 by Heyting, etc. So, 75 years before Atanassov used it, it already had a specific meaning in logic. Moreover, one year after Atanassov's first use of it in Bulgarian, Takeuti and Titani (1984) (see [135]) presented a set representation for Heyting ideas, using the expression intuitionistic fuzzy sets. From our point of view, this means that in fact the correct terminology is that of Takeuti and Titani. Nevertheless, all these facts have originated a serious notation problem in the literature about the subject. To solve this problem, several different solutions have been proposed:

1) To use the notation AIFSs; that is, Atanassov intuitionistic fuzzy sets. However, Atanassov himself asserts that his original naming must be kept [7].

2) To use the name of IF-sets [67].

3) To use the name Bipolar Fuzzy Sets. In particular, in 2005 in [54], the following sentence appears: in fact, since the term bipolarity seems to be agreed upon in some communities, from preference modelling to cognitive psychology, as capturing the separate handling of positive and negative aspects of information, one may suggest a more radical change and call IFSs bipolar fuzzy sets. However, it is important to say that, in the fuzzy setting, with the word bipolar we have the same problem as with the word intuitionistic, since the term bipolarity was used in the fuzzy setting by Zhang [166] and Lee [84], eight and five years, respectively, before the proposal in 2005.

4.- Applications. Extensions have shown to be very useful in problems of decision making [55], [85], [112], [152], [153], [154], [159], [169], [170]. In general, they work very well in problems in which we have to represent the difference between the positive and the negative representation of something [37], in particular in cognitive psychology and medicine [19]. Also in image processing they have been used often, as in [20], [89]. We should remark that the mathematical identity between these sets and IVFSs makes that, in many applications in which IVFSs are useful, so are AIFSs [41].

\section{INTERVAL-VALUED ATANASSOV INTUITIONISTIC FUZZY SETS}

\section{A. Basic Definitions}

IVFSs and AIFSs can be extended to a more general framework to simultaneously deal with uncertainty in both membership and non-membership values. This consideration leads to the concept of Interval-Valued AIFS (IVAIFS), as given by Atanassov and Gargov in 1989 [6].

Definition 9.1: An IVAIFS $A$ on $X$ is a mapping

$$
\begin{aligned}
& A: X \rightarrow \operatorname{LL}([0,1])=\{([\underline{\mu}, \bar{\mu}],[\underline{\nu}, \bar{\nu}\}]) \mid \\
& \quad[\underline{\mu}, \bar{\mu}],[\underline{\nu}, \bar{\nu}] \in L([0,1]) \text { such that } \bar{\mu}+\bar{\nu} \leq 1\} .
\end{aligned}
$$

Recall that $L([0,1])$ denotes the set of all closed subintervals of the unit interval.
In Definition 9.1, IVAIFSs are adapted to Zadeh's ideas on the problem of building the membership degrees of the elements to the FS. Moreover, if for every $x \in X$ we have that $\underline{\mu}(x)=\bar{\mu}(x)$ and $\underline{\nu}(x)=\bar{\nu}(x)$, then we recover an AIFS, so the later are a particular case of IVAIFSs. As in the case of AIFSs, the complement of a set is obtained by interchanging the membership and non-membership intervals.

We represent by $\operatorname{IVAIFS}(X)$ the class of all IVAIFS over a universe $X$.

Definition 9.2: Given $A, B \in \operatorname{IVAIFS}(X)$, we define

$$
A \cup_{\text {IVAifs }} B=\left\{\left(x, A \cup_{\text {IVAifs }} B(x)\right) \mid x \in X\right\}
$$

where

\section{$A \cup_{\text {IVAIFS }} B(x)=$}

$$
\begin{aligned}
& \left(\left[\max \left(\underline{\mu_{A}}(x), \underline{\mu_{B}}(x)\right), \max \left(\overline{\mu_{A}}(x), \overline{\mu_{B}}(x)\right)\right]\right. \\
& \left.\left[\min \left(\underline{\nu_{A}}(x), \underline{\nu_{B}}(x)\right), \min \left(\overline{\nu_{A}}(x), \overline{\nu_{B}}(x)\right)\right]\right)
\end{aligned}
$$

and

$$
A \cap_{\text {IVaifs }} B=\left\{\left(x, A \cap_{\text {IVaifs }} B(x)\right) \mid x \in X\right\},
$$

where

$$
\begin{aligned}
A \cap_{\text {IVAifs }} B(x) & = \\
& \left(\left[\min \left(\underline{\mu_{A}}(x), \underline{\mu_{B}}(x)\right), \min \left(\overline{\mu_{A}}(x), \overline{\mu_{B}}(x)\right)\right]\right. \\
& {\left.\left[\max \left(\underline{\nu_{A}}(x), \underline{\nu_{B}}(x)\right), \max \left(\overline{\nu_{A}}(x), \overline{\nu_{B}}(x)\right)\right]\right) . }
\end{aligned}
$$

Note that $\operatorname{LL}([0,1])$ with the operations in Definition 9.2 is a lattice. Consequently, IVAIFSs are a particular case of L-fuzzy sets.

Proposition 9.1: The set (IVAIFS $\left.(X), \cup_{\text {IVAIFS }}, \cap_{\text {IVAIfS }}\right)$ is a complete bounded lattice.

\section{B. IVAIFSs are a Special Case of HFSs}

In general, the membership degree $\left(\left[\mu_{A}(x), \overline{\mu_{A}}(x)\right]\right)$ and the non-membership degree $\left(\left[\nu_{A}(x), \overline{\nu_{A}}(x)\right]\right)$ may overlap and, hence, the operations in Eq. (12) cannot be applied directly in order to consider the HFS structure. To solve this problem, intuitively it is necessary to found a procedure which separates the membership interval from the non-membership interval. This can be done in many ways, just by choosing any function which maps membership intervals and non-membership intervals into two disjoint subsets of $[0,1]$. For instance, if considering

$$
\begin{aligned}
\underline{j}: L([0,1]) & \rightarrow L([0,1]) \\
{[\underline{x}, \bar{x}] } & \mapsto\left[\frac{1}{3} \underline{x}, \frac{1}{3} \bar{x}\right],
\end{aligned}
$$

and

$$
\begin{aligned}
\bar{j}: L([0,1]) \rightarrow & L([0,1]) \\
{[\underline{x}, \bar{x}] \mapsto } & {\left[\frac{1}{3}(1-\bar{x})+\frac{2}{3}, \frac{1}{3}(1-\underline{x})+\frac{2}{3}\right]=} \\
& {\left[1-\frac{1}{3} \bar{x}, 1-\frac{1}{3} \underline{x}\right], }
\end{aligned}
$$

then we have the following result.

Proposition 9.2: IVAIFSs are a particular case of HFSs. 
Proof. Consider the mapping that assigns, to each IVAIFS $A$, the set

$$
h_{A}(x)=\underline{j}\left(\left[\underline{\mu_{A}}(x), \overline{\mu_{A}}(x)\right]\right) \cup \bar{j}\left(\left[\underline{\nu_{A}}(x), \overline{\nu_{A}}(x)\right]\right) .
$$

This mapping defines a bijection between the class of IVAIFSs and that of HFSs such that, for every $x \in X, h_{A}(x)$ belongs to $\left\{[a, b] \cup[c, d] \mid 0 \leq a \leq b \leq \frac{1}{3}\right.$ and $\left.\frac{2}{3} \leq c \leq d \leq 1, c-b=\frac{2}{3}\right\}$. The result follows.

Remark 4: Note that the choice of the factor $\frac{1}{3}$ for the definition of $\underline{j}$ and $\bar{j}$ is arbitrary. In fact, any value $\delta \in] 0, \frac{1}{2}[$ also works, since the only relevant aspect is that the membership interval and the non-membership interval for each element are transformed into intervals in $L([0,1]$ that are disjoint.

With this definition, for any two interval-valued AIFSs, it holds that

$$
\begin{aligned}
& h_{A \cup_{\mathrm{HF}} B}(x)= \\
& {\left[\max \left(\frac{1}{3} \underline{\mu_{A}}(x), \frac{1}{3} \underline{\mu_{B}}(x)\right), \max \left(\frac{1}{3} \overline{\mu_{A}}(x), \frac{1}{3} \overline{\mu_{B}}(x)\right)\right] \cup} \\
& {\left[\min \left(1-\frac{1}{3} \overline{\nu_{A}}(x), 1-\frac{1}{3} \overline{\overline{\nu_{B}}}(x)\right),\right.} \\
& \left.\quad \max \left(1-\frac{1}{3} \underline{\nu_{A}}(x), 1-\frac{1}{3} \underline{\nu_{B}}(x)\right)\right]= \\
& {\left[\max \left(\frac{1}{3} \underline{\mu_{A}}(x), \frac{1}{3} \underline{\mu_{B}}(x)\right), \max \left(\frac{1}{3} \overline{\mu_{A}}(x), \frac{1}{3} \overline{\mu_{B}}(x)\right)\right] \cup} \\
& {\left[\min \left(\underline{\nu_{A}}(x), \underline{\nu_{B}}(x)\right), \max \left(\overline{\nu_{A}}(x), \overline{\nu_{B}}(x)\right] .\right.}
\end{aligned}
$$

Hence the classical union and intersection operations between IVAIFSs are recovered.

\section{Some Considerations about IVAIFSs}

Some important aspects about IVAIFSs have to be considered.

1.- Measures as pairs of intervals. It is necessary to study two different types of information measures: those whose outcome is a single number [24] and those whose outcome are two intervals in $[0,1]$.

2.- Applications. Nowadays there are several works using these sets [87], [148], [155], [160]. However, none of them shows an example where the results obtained with these sets are better than those obtained with FSs or other techniques. As it happened until recent years with IVFSs, it is necessary to find an application that provides better results using these extensions rather than using other sets. To do so, results with IVAIFSs should be confronted to those obtained with other extensions, which is something that it is not done so far. For the moment, most of the studies are merely theoretical ([8], [57], [145]).

\section{Fuzzy Multisets and n-Dimensional Fuzzy Sets}

Besides, the idea of a fuzzy multiset (MS) was given by Yager in 1986 in [156] and later developed by Miyamoto in [105]. In these multilevel sets, several degrees of membership are assigned to each element.
Definition 10.1: Let $n \in \mathbb{N}_{0}$. A MS $A$ on the universe $X$ is a mapping

$$
A: X \rightarrow[0,1]^{n} .
$$

If, in Definition 10.1, we impose $u_{1} \leq u_{2} \leq \cdots \leq u_{n}$, where $A(x)=\left(u_{1}, \ldots, u_{n}\right)$, an $n$-dimensional fuzzy set is obtained [13], [131]. Nevertheless, it is worth to point out the relation of these families of fuzzy sets with the classification model proposed in [3], and the particular model proposed in [107], where fuzzy preference intensity was arranged according to the basic preference attitudes.

Proposition 10.1: MSs are a particular case of SVFSs.

\section{Four Types of Special Sets with a Fuzzy Set GENERALIZATION}

In this section three types of sets are recalled. They are treated differently from the ones in previous sections because the way in which they deal with uncertainty is intrinsically different. For instance, rough set theory is a tool for the analysis of indistinguibility of objects/classes [114]. In [114] it is argued that rough sets, even dealing with uncertainty, can not be compared to FSs, since they are measuring a different kind of uncertainty. We leave for the future the analysis of the relations between the four types of sets considered in this secion an the other types of fuzzy sets that we have analyzed in the work.

\section{A. Fuzzy Rough Sets}

From the concept of rough set given by Pawlak in [113], Dubois and Prade [50] propose the following definition.

Definition 11.1: Consider a universe $X$ and let $R$ be a fuzzy similarity relation on $X$. Let $A \in \mathrm{FS}(X)$. A fuzzy rough set on $X$ is a pair $(R \downarrow A, R \uparrow A) \in \operatorname{FS}(X) \times \operatorname{FS}(X)$ where

(i) $R \downarrow A: X \rightarrow[0,1]$ is given by $R \downarrow A(x)=$ $\inf _{u \in X} \max (1-R(u, x), A(u))$,

(ii) $R \uparrow A: X \rightarrow[0,1]$ is given by $R \uparrow A(x)=$ $\sup _{u \in X} \min (R(u, x), A(u))$.

These sets can be considered as a generalization of FSs in our sense. They have been widely used in the literature, for example in data reduction (feature selection, instance selection, ...) [142], [44].

\section{B. Fuzzy Soft Sets}

Based on the definition of a soft set [12], [106], Maji et al. presented [88] the following definition.

Definition 11.2: A pair $(F, A)$ is called a fuzzy soft set (FSS) over $X$, where $F$ is a mapping given by $F: A \rightarrow$ $\operatorname{FS}(X)$, where $\operatorname{FS}(X)$ denotes the set of all fuzzy subsets of $X$ and $A$ is a set of parameters.

\section{C. m-Polar-Valued Fuzzy Sets}

The idea of bipolarity is related to the existence of two poles, but can be generalized to consider any arbitrary number. This point of view leads to introduce the notion of a multi-polar-valued fuzzy set. This concept may be defined as follows. 
Definition 11.3: [100] Let $m \geq 2$. An $m$-polar-valued fuzzy set (mPVFS) on $X$ is a mapping

$$
A: X \rightarrow\{1, \ldots, m\} \times[0,1] .
$$

Note that this type of sets extends the BVFSs. Note also that the semantics becomes much more complicated, in particular because a given pole is not necessarily opposite to any other one. From a formal point of view, given two $m$-polar-valued fuzzy sets $A$ and $B$, their union is defined as [101], [102], [103]:

$A \cup B=\left\{\left(x,\left(\left(k, \max \left(\pi_{k}\left(A(x), \pi_{k}(B(x))\right)\right)\right)_{k=1}^{m}\right) \mid x \in X\right\}\right.$, while their intersection is defined as

$A \cap B=\left\{\left(x,\left(\left(k, \min \left(\pi_{k}\left(A(x), \pi_{k}(B(x))\right)\right)\right)_{k=1}^{m}\right) \mid x \in X\right\}\right.$, where, if $(x)=\left\{\left(1, t_{1}\right), \ldots,\left(m, t_{m}\right)\right\}, t_{1}, \ldots, t_{m} \in[0,1]$, then $\pi_{k}(A(x))=t_{k}$. From a mathematical point of view, $\{1, \ldots, m\} \times[0,1]$ is equivalent to $[0,1]^{m}$, and a bounded lattice structure is recovered.

\section{Complex fuzzy sets}

In 2002, Ramot et al. [119] proposed the idea of a complex fuzzy set (CFS) (which was later also developed by Dick [47]) as follows.

Definition 11.4: A CFS A on the universe $X$ is a mapping

$$
A: X \rightarrow \mathbb{D}
$$

where

$$
\mathbb{D}=\left\{r e^{i s} \mid r, s \in[0,1] \text { and } i=\sqrt{-1}\right\}
$$

That is, the membership function of a CFS $A$ takes its values from the unit disk in the complex plane. Note that, in this way, FSs are just a particular case of CFSs.

\section{CONCLUSIONS}

In this work we have introduced the definition and the basic properties of different types of FSs that can be found in the literature. We have also analyzed their relationships. In particular, we have proved that the notion of a hesitant fuzzy set is the same as the notion of a set-valued fuzzy set as introduced by Grattan-Guinness in 1976, although the former is equipped with union and intersection operations that allow to extend the union and intersection defined by Zadeh for FS. We have also proved that Atanassov intuitionistic fuzzy sets, vague sets, grey sets, interval-valued fuzzy sets, intervalvalued Atanassov intuitionistic fuzzy sets and some types of bipolar sets are particular cases of set-valued fuzzy sets. Moreover, we have also shown that the original mathematical formulation of interval type-2 fuzzy set also corresponds to a set-valued fuzzy set.

As said before, there exist different ways to handle uncertainty and imperfect information. Nowadays, there exist in the literature several combinations of the different types of fuzzy sets considered up to now. For instance, IVFSs and soft sets in [144], AIFSs and rough sets [75], IVFSs and HFSs [38], [118], soft sets and HFSs [143] and many other combinations.

\section{Acknowledgments}

This work has been supported by projects TIN2013-40765$P$ and TIN2012-32482 of the Spanish Ministry of Science and by the Brazilian funding agency CNPq (Conselho Nacional de Desenvolvimento Científico e Tecnológico), under Proc. No. 480832/2011-0 and No. 307681/2012-2.

\section{REFERENCES}

[1] Aisbett, J., Rickard, J.T, Morgenthaler, D.G., Type-2 fuzzy sets as functions on spaces, IEEE Transactions on Fuzzy Systems, 18(4) (2010) 841-844.

[2] Alcalá-Fernandez, J., Alcalá, R., Herrera, F., A fuzzy association rulebased classification model for high-dimensional problems with genetic rule selection and lateral tuning, IEEE Transactions on Fuzzy Systems, 19(5) (2011) 857-872.

[3] Amo, A., Montero, J., Biging, G., Cutello, V., Fuzzy classification systems, European Journal of Operational Research 156 (2004) 459-507.

[4] Atanassov, K.T., Intuitionistic fuzzy sets, VII ITKR's Session, Sofia (deposited in Central Science-Technical Library of Bulgarian Academy of Science, (1983) 1697-1684 (in Bulgarian).

[5] Atanassov, K.T., Intuitionistic fuzzy sets, Fuzzy Sets and Systems 20 (1986) 87-96.

[6] Atanassov, K.T., Gargov, G., Interval valued intuitionistic fuzzy sets, Fuzzy Sets and Systems, 31(3), (1989) 343-349.

[7] Atanassov, K.T., Answer to D. Dubois, S. Gottwald, P. Hajek, J. Kacprzyk and H. Prade's paper Terminological difficulties in fuzzy set theory - the case of "Intuitionistic fuzzy sets", Fuzzy Sets and Systems, 156(3) (2005) 496-499.

[8] Aygunoglu, A.; Varol, B. Pazar; Cetkin, V. Aygun, H., Interval-valued intuitionistic fuzzy subgroups based on interval-valued double t-norm, Neural Computing and Applications, 21(1) (2012) S207-S214.

[9] Baccour, L., Alimi, A.M., John, R. I., Similarity measures for intuitionistic fuzzy sets: State of the art, Journal of Intelligent and Fuzzy Systems, 24(1) (2013) 37-49.

[10] Bandler, W., Kohout, L., Fuzzy power sets, fuzzy implication operators, Fuzzy Sets and Systems, 4 (1980) 13-30

[11] Barrenechea, E., Bustince, H., De Baets, B., Lopez-Molina, C., Construction of interval-valued fuzzy relations with application to the generation of fuzzy edge images, IEEE Transactions on Fuzzy Systems, 19(5) (2011) 819-830.

[12] Basu, K., Deb, R., Pattanail, P. K., Soft sets: an ordinal formulation of vagueness with some applications to the theory of choice, Fuzzy Sets and Systems, 45 (1992) 45-58.

[13] Bedregal, B., Beliakov, G., Bustince, H., Calvo, T., Mesiar, R., Paternain, D., A class of fuzzy multisets with a fixed number of memberships, Information Sciences, 189 (2012) 1-17.

[14] Bedregal, B., Reiser, R., Bustince, H. Lopez-Molina, C. Torra, V., Aggregation functions for typical hesitant fuzzy elements and the action of automorphisms, Information Sciences, 255(10) (2014) 82-99.

[15] Bedregal, B., Santiago, R., Interval representations, Łukasiewicz implicators and Smets-Magrez axioms, Information Sciences, 221(10) (2013) 192-200.

[16] Bedregal, B., Santiago, R., Bustince, H., Paternain, D., Reiser, R., Typical hesitant fuzzy negations, International Journal of Intelligent Systems, 26(6) (2014) 525-543.

[17] Beliakov, G., Pagola, M., Wilkin, T., Vector valued similarity measures for Atanassov's intuitionistic fuzzy sets, Information Sciences, 280 (2014) 352-367.

[18] Bellman, R.E., Kalaba, R., Zadeh, L.A., Abstraction and pattern classification, Journal of Mathematical Analysis and Applications, 13 (1966) $1-7$.

[19] Biswas, S.K., Roy, A.R., An application of intuitionistic fuzzy sets in medical diagnosis, Fuzzy Sets and Systems 117 (2001) 209-213.

[20] Bloch, I., Lattices of fuzzy sets and bipolar fuzzy sets, and mathematical morphology, Information Sciences, 181(10) (2011) 2002-2015.

[21] Burillo, P., Bustince, H., Entropy on intuitionistic fuzzy sets and on interval-valued fuzzy sets, Fuzzy Sets and Systems 78 (1996) 305-316.

[22] Bustince, H., Barrenechea, E., Fernandez, J., Pagola, M., Montero, J., The origin of fuzzy extensions, in: J. Kacprzyk, W.Pedrycz (Ed.), Handbook of Computational Intelligence, Springer, 2015.

[23] Bustince, H., Burillo, P., Vague sets are intuitionistic fuzzy sets, Fuzzy Sets and Systems, 79(3) (1996) 403-405.

[24] Bustince, H., Burillo, P., Correlation of interval-valued intuitionistic fuzzy sets, Fuzzy Sets and Systems, 74(2) (1995) 237-244. 
[25] Bustince, H., Herrera, F., Montero, J., Fuzzy Sets and Their Extensions: Representation, Aggregation and Models, Springer, Berlin, 2007.

[26] Bustince, H., Fernandez, J., Hagras, H., Herrera, F., Pagola, M., Barrenechea, E., Interval Type-2 Fuzzy Sets are generalization of IVFSs: Towards a Wider view on their relationship, in Press IEEE Transactions on Fuzzy Systems DOI: 10.1109/TFUZZ.2014.2362149.

[27] Bustince, H., Montero, J., Pagola, M., Barrenechea, E., Gómez, D. A survey of interval-valued fuzzy sets, in:W.Pedrycz (Ed.), Handbook of Granular Computing,Wiley, New Jersey, 2008.

[28] Bustince, H., Montero, J., Barrenechea, E., Pagola, M. , Semiautoduality in a restricted family of aggregation operators, Fuzzy Sets and Systems, 158 (12) 2007, 1360-1377.

[29] Bustince, H., Galar M., Bedregal B., Kolesárová, A., Mesiar, M., A new approach to interval-valued Choquet integrals and the problem of ordering in IVFSs applications, IEEE Transactions on Fuzzy Systems, 21(6) (2013) 1150-1162.

[30] Bustince, H., Barrenechea, Pagola, M., Fernandez, J., Sanz, J., Image thresholding using type II fuzzy sets. Importance of this method, Pattern Recognition, 43(9) (2010) 3188-3192.

[31] Bustince, H., Barrenechea, E., Pagola, M., Fernandez, J., Interval valued fuzzy sets constructed from matrices: Application to edge detection, Fuzzy Sets and Systems, 60(13) (2009) 1819-1840.

[32] Bustince, H., Pagola, M., Barrenechea, E., Fernandez, J., Melo-Pinto, P., Couto, P., Tizhoosh, H.R., Montero, J., Ignorance functions. An application to the calculation of the threshold in prostate ultrasound images, Fuzzy Sets and Systems, 161 (1) (2010) 20-36.

[33] Bustince, H., Fernandez, J., Kolesárová, A., Mesiar, R., Generation of linear orders for intervals by means of aggregation functions, Fuzzy Sets and Systems, 220 (2013) 69-77.

[34] Calvo, T., Kolesárová, A., Komorníková, M., Mesiar, R., Aggregation operators: properties, classes and construction methods. In T. Calvo, G. Mayor and R. Mesiar (Eds.): Aggregation Operators New Trends and Applications (Physica-Verlag, Heidelberg); Pages 3-104, 2002.

[35] Castillo, O., Melin, P., A review on interval type-2 fuzzy logic applications in intelligent control. Information Sciences 279 (2014) 615-631.

[36] Chen, S.M., Wang, H. Y., Evaluating students answer scripts based on interval-valued fuzzy grade sheets, Expert Systems with Applications, 36(6) (2009) 9839-9846.

[37] Chen, T., Multi-criteria decision-making methods with optimism and pessimism based on Atanassov's intuitionistic fuzzy sets, International Journal of Systems Science, 43(5) (2012) 920-938.

[38] Chen, N., Xu, Z.S., Xia, M. M., Interval-valued hesitant preference relations and their applications to group decision making, KnowledgeBased Systems, 37(1) (2013) 528-540.

[39] Choi, B.I., Rhee, F.C.H, Interval type-2 fuzzy membership function generation methods for pattern recognition, Information Sciences, 179, (13) (2009) 2102-2122.

[40] Choi, H.M., Mun, G. S., Ahn, J.Y., A medical diagnosis based on interval-valued fuzzy sets, Biomedical Engineering-Applications Basis Communications, 24(4) (2012) 349-354.

[41] Couto, P., Jurio, A., Varejao, A., Pagola, M., Bustince, H., Melo-Pinto, P., An IVFS-based image segmentation methodology for rat gait analysis, Soft Computing, 15(10) (2011) 1937-1944.

[42] De Baets, B., Kerre, E.E., Gupta, M., The fundamentals of fuzzy mathematical morphology part 1: Basic Concepts, International Journal of General Systems, 23(2) (1995) 155-171.

[43] Deng, J.L., Introduction to grey system theory, Journal of Grey Systems 1 (1989) 1-24.

[44] Derrac, J., Cornelis, C., García, S., Herrera, F., Enhancing evolutionary instance selection algorithms by means of fuzzy rough set based feature selection, Information Sciences, 186(1) (2012) 73-92

[45] Deschrijver, G. Kerre, E.E., On the position of intuitionistic fuzzy set theory in the framework of theories modelling imprecision, Information Sciences, 177 (2007) 1860-1866.

[46] Deschrijver, G., Kerre, E.E., On the relationship between some extensions of fuzzy set theory, Fuzzy Sets and Systems, 133 (2003) 227-235.

[47] Dick, S., Toward complex fuzzy logic, IEEE Transactions on Fuzzy Systems, 13 (2005) 405-414.

[48] Dubois, D., Prade, H., Fuzzy sets and systems: Theory and applications, Academic Press, 1980.

[49] Dubois, D., Prade, H., Operations in a fuzzy-valued logic, Information \& Control, 43(2) (1979) 224-254.

[50] Dubois, D., Prade, H., Rough fuzzy sets and fuzzy rough sets, International Journal of General Systems, 17 (1990) 191-209.

[51] Dubois, D., Prade, H., Are grey sets novel?, BUSEFAL, 44 (1990) 138141.
[52] Dubois, D., Prade, H., An introduction to bipolar representations of information and preference. International Journal of Intelligent Systems, 23 (2008) 866-877.

[53] Dubois, D., Prade, H., An overview of the asymmetric bipolar representation of positive and negative information in possibility theory, Fuzzy sets and Systems, 160(10) (2009) 1355-1366.

[54] Dubois, D., Gottwald, S., Hajek, P., Kacprzyk, J., Prade, H., Terminological difficulties in fuzzy set theory.The case of Intuitionistic Fuzzy Sets, Fuzzy Sets and Systems, 156(3) (2005) 485-491.

[55] Dudziak, U., Pekala, B., Equivalent bipolar fuzzy relations, Fuzzy Sets and Systems, 161(2) (2010) 234-253.

[56] Dziech, A., Gorzalczany, M.B. Decision making in signal transmission problems with interval-valued fuzzy sets, Fuzzy Sets and Systems 23(2) (1987) 191-203.

[57] Fanyong, M., Qiang, Z., Hao. Ch., Approaches to multiple-criteria group decision making based on interval-valued intuitionistic fuzzy Choquet integral with respect to the generalized lambda-Shapley index, Knowledge-Based Systems, 37 (2013) 237-249.

[58] Fodor, J., Roubens, M., Fuzzy Preference Modelling and Multicriteria Decision Support, in: Theory and Decision Library (Kluwer Academic Publishers) 1994.

[59] Galar, M., Fernandez, F., Beliakov, G., Bustince, H., Interval-valued fuzzy sets applied to stereo matching of color images. IEEE Transactions on Image Processing 20 (2011) 1949-1961.

[60] Gau, W.L., Buehrer, D.J., Vague sets, IEEE Transactions on Systems Man and Cybernetics. 23(2)(1993) 610-614

[61] Goguen, J. L-fuzzy sets, Journal of Mathematical Analysis and Applications 18 (1967) 145-174.

[62] Gorzalczany, M.B., A method of inference in approximate reasoning based on interval-valued fuzzy sets, Fuzzy Sets and Systems 21 (1987) 1-17.

[63] Gorzalczany,M.B., Interval-valued fuzzy controller based on verbal model of object, Fuzzy Sets and Systems 28(1) (1988) 45-53.

[64] Gorzalczany, M.B. Interval-valued fuzzy inference involving uncertain (inconsistent) conditional propositions, Fuzzy Sets and Systems 29(2) (1989) 235-240.

[65] Gorzalczany,M.B., An interval-valued fuzzy inference method. Some basic properties, Fuzzy Sets and Systems 31(2) (1989) 243-251.

[66] Grattan-Guinness, I., Fuzzy membership mapped onto interval and many-valued quantities, Zeitschrift für mathematische Logik und Grundladen der Mathematik 22 (1976) 149-160.

[67] Grzegorzewski, P., On some basic concepts in probability of IF-events, Information Science, 232 (2013) 411-418.

[68] Hagras, H., A hierarchical type-2 fuzzy logic control architecture for autonomous mobile robots, IEEE Transactions on Fuzzy Systems 12 (2004) 524-539.

[69] Hagras, H., Wagner, C., Towards the wide spread use of type-2 fuzzy logic systems in real world applications, IEEE Computational Intelligence Magazine 7 (3) (2012) 14-24

[70] Harding, J., Walker, C., Walker, E., The variety generated by the truth value algebra of T2FSs, Fuzzy Sets and Systems, 161 (2010) 735-749.

[71] Hernández, P.R., Contribución al estudio de las negaciones, autocontradicción, t-normas y t-conormas en los conjuntos borrosos de tipo 2, Ph.D. thesis (in Spanish), Universidad Politécnica de Madrid, Junio 2014.

[72] Hernández, P.R., Cubillo, S., Torres-Blanc, C., Negations on type-2 fuzzy sets, Fuzzy Sets and Systems, 252 (2014) 111-124.

[73] Hernández, P.R., Cubillo, S., Torres-Blanc, C., On t-norms for type-2 fuzzy sets, IEEE Transactions on Fuzzy Systems, (2014) In press, doi: 10.1109 / TFUZZ.2014.2346247.

[74] Herrera, F., Herrera-Viedma, E., Linguistic decision analysis: steps for solving decision problems under linguistic information. Fuzzy Sets and Systems 115 (2000) 67-82.

[75] Huang, B., Guo, C.-X. , Zhuang, Y.-L., Li, H.-X. , Zhou, X.-Z., Intuitionistic fuzzy multigranulation rough sets, Information Sciences, 277 (2014) 299-320.

[76] Huang, L.K., Wang, M.J., Image thresholding by minimizing the measure of fuzziness, Pattern Recognition, 29(1) (1995) 41-51.

[77] Huhn, J., Hüllermeier, E., FURIA: an algorithm for unordered fuzzy rule induction, Data Mining and Knowledge Discovery 19(3) (2009) 293-319.

[78] Jahn, K.U., Intervall-wertige Mengen, Mathematische Nachrichten 68 (1975) 115-132.

[79] Jurio, A., Pagola, M.,Paternain, D., Lopez-Molina, C., Melo-Pinto, P., Interval-valued restricted equivalence functions applied on Clustering Techniques in Proceedings of the Joint 2009 International Fuzzy Systems Association World Congress and 2009 European Society of Fuzzy Logic and Technology, 2009, 831-836. 
[80] Karnik, N.N., Mendel, J.M., Introduction to type-2 fuzzy logic systems, IEEE FUZZ Conference, Anchorage, AK, May 1998

[81] Karnik, N.N., Mendel, J.M., Liang, Q., Type-2 Fuzzy Logic Systems, IEEE Transactions on Fuzzy Systems on Fuzzy Systems, 7 (1999) 643658.

[82] Klement, E.P., Mesiar, R., Pap, E., Triangular Norms, Trends in Logic. Studia Logica Library, Kluwer Academic Publishers, Dordrecht, 2000.

[83] Klir, G.J., Yuan, B., Fuzzy sets and fuzzy logic: theory and applications, Prentice-Hall, 1995.

[84] Lee, K.M., Bipolar-valued fuzzy sets and their operations, Conference on Intelligent Technologies, Bangkok, Thailand (2000), 307-312.

[85] Li, B., He, W., Intuitionistic fuzzy PRI-AND and PRI-OR aggregation operators, Information Fusion, 14 (2013) 450-459

[86] Liu, F., Geng, H. , Zhang,Y.-Q., Interactive fuzzy interval reasoning for smart web shopping, Applied Soft Computing 5(4) (2005) 433-439.

[87] Liu, B., Shen, Y., Chen, X., Chen, Y., Wang, X., A partial binary tree DEA-DA cyclic classification model for decision makers in complex multi-attribute large-group interval-valued intuitionistic fuzzy decisionmaking problems, Information Fusion, 18 (2014) 119-130.

[88] Maji, P.K., Biswas, R., Roy, R., Fuzzy soft sets, The Journal of Fuzzy Mathematics, 9(3) (2001) 589-602.

[89] Melo-Pinto, P., Couto, P., Bustince, H., Barrenechea, E., Pagola, M., Fernandez, J., Image segmentation using Atanassov's intuitionistic fuzzy sets, Expert Systems with Applications, 40(1) (2013) 15-26.

[90] Mendel, J.M., Advances in Type-2 fuzzy sets and systems, Information Sciences 177 (2007) 84-110.

[91] Mendel, J.M., Uncertain Rule-Based Fuzzy Logic Systems: Introduction and New Directions, Upper Saddle River, NJ: Prentice-Hall, 2001.

[92] Mendel, J. M., Hagras, H., Tan, W.-W., Melek, W. W., Ying, H., Introduction to Type-2 Fuzzy Logic Control, John Wiley and IEEE Press, Hoboken, NJ, 2014.

[93] Mendel, J.M., John, R.I., Type-2 fuzzy sets made simple, IEEE Transactions on Fuzzy Systems, 10 (2002) 117-127.

[94] Mendel, J. M.; John, R. I., Liu, F., Interval type-2 fuzzy logic systems made simple, IEEE Transactions on Fuzzy Systems, 14(6) (2006) 808821.

[95] Mendel, J.M., Type-2 fuzzy sets for computing with words, IEEE International Conference on Granular Computing, MAY 10-12, 2006 Atlanta, (2006) GA 8-8.

[96] Mendel, J.M., Computing with words and its relationships with fuzzistics, Information Sciences 177(4) (2007) 988-1006.

[97] Mendel, J.M., Wu,H., Type-2 Fuzzistics for symmetric interval type2 fuzzy sets: Part 1, forward problems, IEEE Transactions on Fuzzy Systems 14(6) (2006) 781-792.

[98] Mendel, J. M., Wu, D., Perceptual Computing: Aiding People in Making Subjective Judgments, John Wiley and IEEE Press, Hoboken, NJ, 2010.

[99] Mendel, J. M., Computing with words: Zadeh, Turing, Popper and Occam, IEEE Computational Intelligence Magazine, 2, (2007) 10-17.

[100] Mesiarová-Zemanková, A., Ahmad, K., Extended multi-polarity and multi-polar-valued fuzzy sets, Fuzzy Sets and Systems, 234 (2014) 6178.

[101] Mesiarová, A., Lazaro, J., Bipolar aggregation operators. Proc. AGOP 2003, Alcalá de Henares, Spain, 2003, 119-123.

[102] Mesiarová-Zemánková, A., Mesiar, R., Ahmad, K., The balancing Choquet inte- gral. Fuzzy Sets and Systems 161 (17) (2010) 2243-2255.

[103] Mesiarová-Zemánková, A., Ahmad, K., Multi-polar Choquet integral, Fuzzy Sets and Systems, 220 (2013) 1-20.

[104] Mizumoto, M., Tanaka, K., Some properties of fuzzy sets of type 2, Information \& Control, 31 (1976) 312-340.

[105] Miyamoto, S., Multisets and fuzzy multisets, in: Z.-Q. Liu, S. Miyamoto (Eds.), Soft Computing and Human-Centered Machines, Springer, Berlin, (2000), 9-33.

[106] Molodtsov, D., Soft set theory first results, Computers and Mathematics with Applications, 37 (1999) 19-31.

[107] Montero, J., Arrow's theorem under fuzzy rationality, Behavioral Science 32 (1987) 267-273.

[108] Montero, J., Gómez, D. Bustince, H., On the relevance of some families of fuzzy sets, Fuzzy Sets and Systems, 158(22), 16 (2007) 2429-2442

[109] Pagola, M., Lopez-Molina, C., Fernandez, J., Barrenechea, E., Bustince, H., Interval type-2 fuzzy sets constructed from several membership functions. Application to the fuzzy thresholding algorithm, IEEE Transactions on Fuzzy Systems, 21(2) (2013) 230-244.

[110] Pal, N.R., Bustince, H, Pagola, M., Mukherjee, U.K., Goswami, D.P., Beliakov, G., Atanassov's intuitionistic fuzzy entropies: Fuzziness and lack of knowledge, Information Sciences, 228 (2013) 61-74.
[111] Palmeira, E.S. , Bedregal, B.,, Extension of fuzzy logic operators defined on bounded lattices via retractions, Computers \& Mathematics with Applications 63(6) (2012) 1026-1038.

[112] Paternain, D., Jurio, A., Barrenechea, E., Bustince, H., Bedregal, B., Szmidt, E., An alternative to fuzzy methods in decision-making problems. Expert Systems with Applications 39(9) (2012) 7729-7735.

[113] Pawlak, Z., Rough sets, International Journal of Computer and Information Sciences 11 (1982) 341-356.

[114] Pawlak, Z., Theoretical Aspects of Reasoning about Data, Kluwer Academic Publishers, Dordrecht, Boston,1991.

[115] Pedrycz, W., Shadowed sets: representing and processing fuzzy sets, IEEE Transactions on Systems Man and Cybernetics, part B 28 (1998) 103-109.

[116] Pedrycz, W., Vukovich, G., Investigating a relevance of fuzzy mappings, IEEE Transactions on Systems Man and Cybernetics, part B 30 (2000) 249-262.

[117] Pedrycz, W., Vukovich, G., Granular computing with shadowed sets, International Journal of Intelligent Systems 17 (2002) 173-197.

[118] Quirós, P., Alonso, P., Bustince, H., Díaz, I., Montes, S, An entropy measure definition for finite interval-valued hesitant fuzzy sets, submitted to Information Sciences.

[119] Ramot, D., Milo, R., Friedman, M., Kandel, A., Complex Fuzzy Sets, IEEE Transactions on Fuzzy Systems, 10 (2002) 171-186.

[120] Rezaei, H., Mukaidono, M., New similarity measures of intuitionistic fuzzy sets, Journal of Advanced Computational Intelligence 203 and Intelligent Informatics 11(2) (2007) 202-209.

[121] Rodriguez, R. M.; Martinez, L.; Herrera, F., Fuzzy linguistic term sets for decision making, IEEE Transactions on Fuzzy Systems, 20(2) (2012) $109-119$.

[122] Rodriguez, R. M.; Martinez, L.; Herrera, F., A group decision making model dealing with comparative linguistic expressions based on hesitant fuzzy linguistic term sets, Information Sciences, 241 (2013) 28-42.

[123] Rodriguez, R. M.; Martinez, L.; Torra, V.; Xu, Z.S.; Herrera, F., Hesitant fuzzy sets: State of the art and future directions, International Journal of Intelligent Systems, 29 (6) (2014) 495-524.

[124] Sambuc, R., Function $\Phi$-Flous, Application a l'aide au Diagnostic en Pathologie Thyroidienne, Thése de Doctorat en Médicine, University of Marseille (1975).

[125] Sanz,J., Bustince, H., Herrera, F., Improving the performance of fuzzy rule-based classification systems with IVFSs and genetic amplitude tuning, Information Sciences 180 (19) (2010) 3674-3685.

[126] Sanz,J., Fernandez, A., Bustince, H., Herrera, F., A genetic tuning to improve the performance of fuzzy rule-based classification systems with IVFSs: Degree of ignorance and lateral position, International Journal of Approximate Reasoning 52 (6) (2011) 751-766.

[127] Sanz, J., Fernandez, A., Bustince, H., Herrera, F., IIVFDT: Ignorance functions based interval-valued fuzzy decision tree with genetic tuning, International Journal of Uncertainty, Fuzziness and Knowledge-Based Systems 20(Suppl. 2) (2012) 1-30.

[128] Sanz, J., Fernandez, A., Bustince, H., Herrera, F., IVTURS: a linguistic fuzzy rule-based classification system based on a new intervalvalued fuzzy reasoning method with Tuning and Rule Selection, IEEE Transactions on Fuzzy Systems, 21(3) (2013) 399-411.

[129] Sanz, J., Galar, M., Jurio, A., Brugos, A., Pagola, M., Bustince, H., Medical diagnosis of cardiovascular diseases using an interval-valued fuzzy rule-based classification system, Applied Soft Computing Journal, 20 (2014) 103-111.

[130] Sanz, J., Bernardo, D., Herrera, F., Bustince, H., Hagras, H., A compact evolutionary interval-valued fuzzy rule-based classification system for the modeling and prediction of real-world financial applications with imbalanced data, IEEE Transactions on Fuzzy Systems, (2014), in Press, doi $=10.1109 /$ TFUZZ.2014.2336263.

[131] Shang, Y., Yuan, X., Lee, E.S., The n-dimensional fuzzy sets and Zadeh fuzzy sets based on the finite valued fuzzy sets, Computers and Mathematics with Applications 60 (2010) 442-463.

[132] Smaradache, F., A unifying field in logics: neutrosophic logic, Multiple-Valued Logic, 8(3) (2002), 385-438.

[133] Szmidt, E., Kacprzyk, J., Bujnowski, P., Measuring the amount of knowledge for Atanassov's intuitionistic fuzzy sets, Lecture Notes in Artificial Intelligence 6857 (2011) 17-24.

[134] Szmidt, E., Kacprzyk, J., Entropy for intuitionistic fuzzy sets, Fuzzy Sets and Systems 118(3) (2001) 467- 477.

[135] Takeuti, G., Titani, S., Intuitionistic fuzzy logic and intuitionistic fuzzy set theory. Journal of Symbolic Logic, 49 (1984) 4851-4866.

[136] Tizhoosh, H.R.,, Image thresholding using type-2 fuzzy sets, Pattern Recognition 38 (2005) 2363-2372. 
[137] Torra, V., Hesitant fuzzy sets, International Journal of Intelligent Systems 25 (2010) 529-539.

[138] Türksen, I. B., Interval valued fuzzy sets based on normal forms, Fuzzy Sets and Systems 20(2) (1986) 191-210.

[139] Türksen, I.B. Interval valued fuzzy sets and compensatory AND, Fuzzy Sets and Systems 51 (1992) 295-307.

[140] Türksen, I.B., Zhong, Z. An approximate analogical reasoning schema based on similarity measures and interval-valued fuzzy sets, Fuzzy Sets and Systems 34 (1990) 323-346.

[141] Türksen, I.B. , Yao, D.D., Representation of connectives in fuzzy reasoning: The view through normal forms, IEEE Transactions on Systems, Man and Cybernetics, 14 (1984) 191-210.

[142] Verbiest, N., Cornelis, C., Herrera, F., FRPS: A fuzzy rough prototype selection method, Pattern Recognition 46(10) (2013) 2770-2782

[143] Wang, F., Li, X., Chen, X., Hesitant fuzzy soft set and its applications in multicriteria decision making, Journal of Applied Mathematics, in press, http://dx.doi.org/10.1155/2014/643785

[144] Wang, J.G., Wu,J.T., Wang, J., Zhang, H.Y., Chen, X.H., Intervalvalued hesitant fuzzy linguistic sets and their applications in multi-criteria decision-making problems, Information Sciences, In Press.

[145] Wang, W, Liu, X., Qin, Y., Interval-valued intuitionistic fuzzy aggregation operators, Journal of Systems Engineering and Electronics, 23(4) (2012) 574-580.

[146] Willmott, R., Mean measures in fuzzy power-set theory, Report No. FRP-6, Department of Mathematics, University of Essex, Colchester, CO4 3SQ, England (1979).

[147] Wu, D.,, Approaches for reducing the computational cost of interval type-2 fuzzy logic systems: Overview and comparisons, IEEE Transactions on Fuzzy Systems, 21(1) (2013) 80-99.

[148] Wu, J., Chiclana, F., Non-dominance and attitudinal prioritisation methods for intuitionistic and interval-valued intuitionistic fuzzy preference relations, Expert Systems with Applications, 39(18) (2012) 1340913416.

[149] Wu, D., Mendel, J. M., A vector similarity measure for linguistic approximation: Interval type-2 and type-1 fuzzy sets, Information Sciences, 178(2) (2008) 381-402

[150] Xia, X. S., Liang, Q. L., Crosslayer design for mobile ad hoc networks using interval type-2 fuzzy logic systems, International Journal of Uncertainty, Fuzziness and Knowledge-Based Systems, 16(3) (2008) 391-408.

[151] Xu, Z.S., Yager, R.R., Some geometric aggregation operators based on intuitionistic fuzzy sets, International Journal of General Systems, 35 (2006) 417-433.

[152] Xu, Z.S., Approaches to multiple attribute group decision making based on intuitionistic fuzzy power aggregation operators, KnowledgeBased Systems, 24(6) (2011) 749-760.

[153] Xu, Z.S., Hu, H., Projection models for intuitionistic fuzzy multiple attribute decision making, International Journal of Information-technology and Decision Making, 9(2) (2010) 267-280.

[154] Xu, Z.S., Priority weights derived from intuitionistic multiplicative preference relations in decision making. IEEE Transactions on Fuzzy Systems , 21(4) (2013) 642-654.

[155] Xu, Z.S., Chen, Q. A multi-criteria decision making procedure based on interval-valued intuitionistic fuzzy Bonferroni means. Journal of Systems Science and Systems Engineering, 20(2) (2011) 217-228.

[156] Yager, R.R., On the theory of bags, International Journal of General Systems 13 (1986) 23-37.

[157] Yager, R.R., Pythagorean fuzzy subsets, IFSA World Congress and NAFIPS Annual Meeting (IFSA/NAFIPS), 2013 Joint (2013) 57-61.

[158] Yager, R.R., Pythagorean membership grades in multicriteria decision making, IEEE Transactions on Fuzzy Systems 22(4) (2014), 958-965.

[159] Ye, J., Fuzzy decision-making method based on the weighted correlation coefficient under intuitionistic fuzzy environment, European Journal of Operational Research, 205(1) (2010) 202-204.

[160] Ye, J., Multicriteria decision-making method using the Dice similarity measure based on the reduct intuitionistic fuzzy sets of interval-valued intuitionistic fuzzy sets, Applied Mathematical Modelling, 36(9) (2012) $4466-4472$

[161] Young R.C., The algebra of many-valued quantities, Mathematische Annalen 104 (1931) 270-290.

[162] Yuksel, M.E., Borlu, M., Accurate segmentation of dermoscopic images by image thresholding based on type-2 fuzzy logic IEEE Transactions on Fuzzy Systems, 17(4) (2009) 976-982.

[163] Zadeh, L.A. Fuzzy Sets, Information \& Control, (1965) 338-353.

[164] Zadeh, L.A. Quantitative fuzzy semantics, Information Sciences 3 (1971) 159-176.

[165] Zadeh, L.A. The concept of a linguistic variable and its application to approximate reasoning-1, Information Sciences, (1975) 199-249.
[166] Zhang,W. R., NPN fuzzy sets and NPN qualitative algebra: A computational framework for bipolar cognitive modeling and multiagent decision analysis. IEEE Transactions on Systems, Man, and Cybernetics-part B: Cybernetics, 26(4) (1996) 561-574.

[167] Zhang, W. R., Zhang, L., Yin Yang bipolar logic and bipolar fuzzy logic. Information Sciences 165 (3-4) (2004) 265-287.

[168] Zhang, W. R. Yin Yang bipolar t-norms and t-conorms as granular neurological operators. Proc. IEEE International Conference on Granular Computing, Atlanta, 91-96.

[169] Zhang, X., Xu, Z.S.,, A new method for ranking intuitionistic fuzzy values and its application in multi-attribute decision making, Fuzzy Optimization and Decision Making, 11(2) (2012) 135-146.

[170] Zhang, Z., Generalized Atanassovs intuitionistic fuzzy power geometric operators and their application to multiple attribute group decision making, Information Fusion, 14 (2013) 460-486

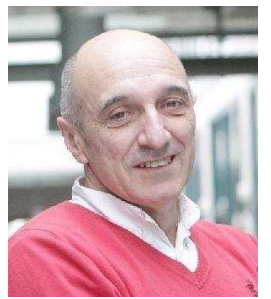

Humberto Bustince (M?08) received the Ph.D. degree in mathematics from the Public University of Navarra, Pamplona, Spain, in 1994. He is currently a Full Professor with the Department of Automatics and Computation, Public University of Navarra. He is the author of more than 65 published original articles and is involved in teaching artificial intelligence for students of computer sciences. His research interests include fuzzy logic theory, extensions of fuzzy sets (type-2 fuzzy sets, intervalvalued fuzzy sets, Atanassovs intuitionistic fuzzy sets), fuzzy measures, aggregation functions, and fuzzy techniques for image processing. Dr. Bustince is a board member of the European Society for Fuzzy Logic and Technology. He is the Editor-in-Chief of the Mathware and Soft Computing Magazine

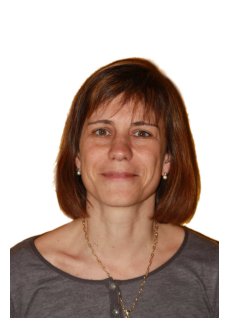

Edurne Barrenechea is an Assistant Lecturer at the Department of Automatics and Computation, Public University of Navarra. She received an M.Sc. in Computer Science at the Pais Vasco University in 1990. She worked in a private company (Bombas Itur) as analyst programmer from 1990 to 2001 , and then she joined the Public University of Navarra as Associate Lecturer. She obtained the Ph.D. in Computer Science in 2005 on the topic intervalvalued fuzzy sets applied to image processing. Her publications comprise more than 30 papers in international journals and about 15 book chapters. Her research interests are fuzzy techniques for image processing, fuzzy sets theory, interval type-2 fuzzy sets theory and applications, decision making, and medical and industrial applications of soft computing techniques. She is member of the board of the European Society for Fuzzy Logic and Technology (EUSFLAT)

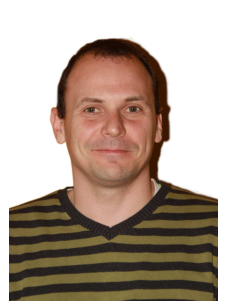

Miguel Pagola received the M.Sc. degree in industrial engineering and the Ph.D. degree, both from the Public University of Navarra (UPNa), Pamplona, Spain, in 2000 and 2008, respectively. He is currently an Associate Lecturer with the Department of Automatics and Computation, UPNa. He is the author or of more than 30 published original articles in the Web of Science and is involved in teaching artificial intelligence for students of computer sciences. His research interests include fuzzy techniques for image processing, fuzzy set theory, clustering and data mining. Dr. Pagola is the member of the European Society for Fuzzy Logic and Technology. 


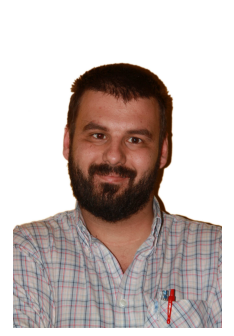

Javier Fernandez received the M.Sc. and Ph.D. degrees in mathematics from the University of Zaragoza, Saragossa, Spain, in 1999 and 2003, respectively. He is currently an Associate Lecturer with the Department of Automatics and Computation, Public University of Navarre, Pamplona, Spain. $\mathrm{He}$ is the author or coauthor of approximately 20 original articles and is involved with teaching artificial intelligence and computational mathematics for students of the computer sciences. His research interests include fuzzy techniques for image processing, fuzzy sets theory, interval-valued fuzzy sets theory, aggregation functions, fuzzy measures, stability, evolution equation, and unique continuation.

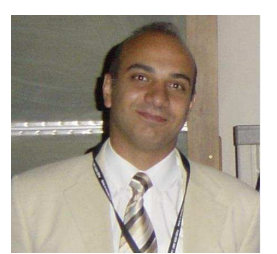

Hani Hagras (M03SM05, F13) He is a Professor in the School of Computer Science and Electronic Engineering, Director of the Computational Intelligence Centre and the Head of the Fuzzy Systems Research Group in the University of Essex, UK. His major research interests are in computational intelligence, notably type-2 fuzzy systems, fuzzy logic, neural networks, genetic algorithms, and evolutionary computation. He has authored more than 300 papers in international journals, conferences and books. He is a Fellow of the Institute of Electrical and Electronics Engineers (IEEE) and he is also a Fellow of the Institution of Engineering and Technology (IET (IEE). His research has won numerous prestigious international awards where most recently he was awarded by the IEEE Computational Intelligence Society (CIS), the 2013 Outstanding Paper Award in the IEEE Transactions on Fuzzy Systems and he was also awarded the 2006 Outstanding Paper Award in the IEEE Transactions on Fuzzy Systems.

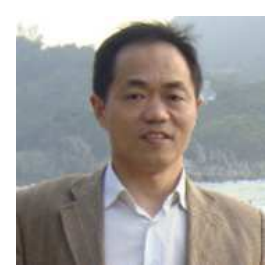

Zeshui Xu (M08-SM09) received the Ph.D. degree in management science and engineering from Southeast University, Nanjing, China, in 2003. He is a Distinguished Young Scholar of the National Natural Science Foundation of China and the Chang Jiang Scholars of the Ministry of Education of China. He is currently a Professor with the Business School, Sichuan University, Chengdu, China. He has contributed more than 400 journal articles to professional journals, and his current research interests include information fusion, decision making, and

fuzzy sets.

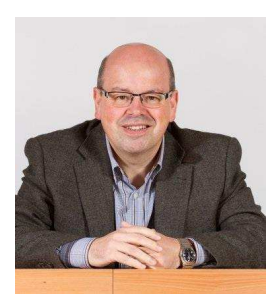

Francisco Herrera received his M.Sc. in Mathematics in 1988 and Ph.D. in Mathematics in 1991, both from the University of Granada, Spain. He is currently a Professor in the Department of Computer Science and Artificial Intelligence at the University of Granada. He has been the supervisor of 37 $\mathrm{Ph} . \mathrm{D}$. students. He received the following honors and awards, among others; ECCAI Fellow 2009, IFSA Fellow 2013, IEEE Transactions on Fuzzy System Outstanding 2008 and 2012 Paper Award. He currently acts as Editor in Chief of the international journals "Information Fusion" (Elsevier) and Progress in Artificial Intelligence (Springer). He acts as editorial member of a dozen of journals. He has published more than 300 papers in international journals. He is coauthor of the books: Genetic Fuzzy Systems: Evolutionary Tuning and Learning of Fuzzy Knowledge Bases" (World Scientific, 2001) and "Data Preprocessing in Data Mining" (Springer, 2015). His current research interests include among others, soft computing (including fuzzy modeling and evolutionary algorithms), information fusion, decision making, bibliometrics, biometric, data preprocessing, data mining, cloud computing and big data.

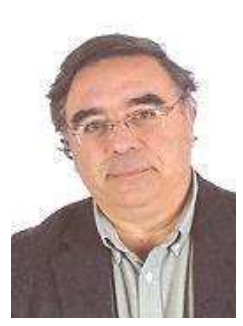

Javier Montero is Full Professor at Complutense University of Madrid. He has been leading research projects since 1987 and has published more than 80 contributions in JCR journals, mainly devoted to the theory and applications of fuzzy sets. $\mathrm{He}$ has served the fuzzy community as President of the European Society for Fuzzy Logic and Technologies (EUSFLAT) and as Vicepresident of the International Fuzzy Systems Association (IFSA). He has been also Dean of the Faculty of Mathematics and Vicerrector at his University

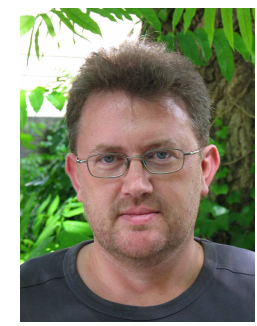

Bernard De Baets (1966) received the MSc degree in mathematics in 1988 , the postgraduate degree in knowledge technology in 1991, and the PhD degree in mathematics in 1995, all summa cum laude from Ghent University, Belgium. He is a full professor at Ghent University, where he is leading the research unit Knowledge-Based Systems (KERMIT). His research focuses on knowledge-based, spatiotemporal, and predictive modeling. 